\title{
Investigating the impact of atmospheric stability on thunderstorm outflow winds and turbulence
}

\author{
Patrick Hawbecker $^{1}$, Sukanta Basu ${ }^{2}$, and Lance Manuel ${ }^{3}$ \\ ${ }^{1}$ Department of Marine, Earth, and Atmospheric Sciences, \\ North Carolina State University, Raleigh, NC 27695, USA \\ ${ }^{2}$ Faculty of Civil Engineering and Geosciences, \\ Delft University of Technology, Delft, the Netherlands \\ ${ }^{3}$ Department of Civil, Architectural, and Environmental Engineering, \\ University of Texas at Austin, Austin, Texas 78712, USA \\ Correspondence: Sukanta Basu (s.basu@tudelft.nl)
}

Received: 5 October 2017 - Discussion started: 17 October 2017

Accepted: 3 April 2018 - Published: 25 April 2018

\begin{abstract}
Downburst events initialized at various hours during the evening transition (ET) period are simulated to determine the effects of ambient stability on the outflow of downburst winds. The simulations are performed using a pseudo-spectral large eddy simulation model at high resolution to capture both the large-scale flow and turbulence characteristics of downburst winds. First, a simulation of the ET is performed to generate realistic initial and boundary conditions for the subsequent downburst simulations. At each hour in the ET, an ensemble of downburst simulations is initialized separately from the ET simulation in which an elevated cooling source within the model domain generates negatively buoyant air to mimic downburst formation.

The simulations show that while the stability regime changes, the ensemble mean of the peak wind speed remains fairly constant (between 35 and $38 \mathrm{~m} \mathrm{~s}^{-1}$ ) and occurs at the lowest model level for each simulation. However, there is a slight increase in intensity and decrease in the spread of the maximum outflow winds as stability increases as well as an increase in the duration over which these strongest winds persist. This appears to be due to the enhanced maintenance of the ring vortex that results from the low-level temperature inversion, increased ambient shear, and a lack of turbulence within the stable cases. Coherent turbulent kinetic energy and wavelet spectral analysis generally show increased energy in the convective cases and that energy increases across all scales as the downburst passes.
\end{abstract}

\section{Introduction}

Severe thunderstorms producing tornadoes and extreme winds have garnered a lot of attention in the wind engineering and wind energy communities due to the high risk of structural damage to buildings and wind turbines caused by these events. In 2016 alone, severe thunderstorms caused an estimated USD 14 billion in insured losses within the United States, roughly $60 \%$ of the total estimated insured losses from natural catastrophes for the year (source: 2017 Munich Re as of February 2017). Historically, there have been several instances of structures directly impacted by these events, resulting in studies focused on estimating the loads generated by these winds on structures such as buildings (Chen, 2008; Sengupta et al., 2008) and electricity transmission towers (Holmes and Oliver, 2000; Oliver et al., 2000; Savory et al., 2001; Chay et al., 2006). With the recent growth of the wind energy industry, the occurrence of severe winds impacting wind turbines has become more common. For example, footage of a tornado moving through a wind farm in Harper County, Kansas, which occurred in 2012 shows the blades of a wind turbine being stripped away and projected (source: https://www.youtube.com/watch?v= Egdtlnv6Gio, last access: 19 April 2018), while a severe thunderstorm event that struck the Buffalo Ridge Wind Farm 
in 2011 ended up causing several wind turbines to lose their blades and the buckling of a tower (Hawbecker et al., 2017). Traditionally, surveys of damage done to features such as buildings, crops, and trees are conducted after an event and, based on the severity of the damage, a rating of 1-5 is assigned based on the Enhanced Fujita (EF) scale (McDonald and Mehta, 2006), formerly known as the Fujita scale (Fujita, 1971, 1981). The EF scale uses damage-wind relationships and bases the ratings on observed damage and design codes. However, indirect estimation of extreme winds based on damage surveys and damage-wind relationships is known to be imprecise and uncertain at times (Reynolds, 1971; Doswell III and Bosart, 2001; Marshall, 2002; Doswell III et al., 2009; Edwards et al., 2013); still, due to the dearth of high-density wind measurement networks, the wind engineering community is compelled to use these estimations. In recent years, high-resolution numerical modeling is gaining momentum as a viable alternative that can serve as a surrogate to in situ data (Dahl et al., 2017).

While high-resolution observations of near-surface winds for severe wind events are rare, climatologies of severe wind events have been developed via several intensive field campaigns and by utilizing storm reports from the National Weather Service (NWS). One such climatology of thunderstorms producing severe winds over the contiguous United States was developed about three decades ago by Kelly et al. (1985). This climatology is generated based on NWS storm reports of thunderstorm-related wind damage over a 29-year period from 1955 through 1983 but it is impossible to distinguish the storm type (i.e., downburst, derecho, rear flank downdraft) from these reports. Around the same time, Wakimoto (1985) generated another storm climatology based solely on observed downbursts from the Joint Airport Weather Studies (JAWS) project (McCarthy et al., 1982). Even though the size of the analyzed dataset was much smaller than that of Kelly et al. (1985), Wakimoto (1985) was able to document the circadian traits of downbursts. Figure 1a shows a regenerated version of the data from Fig. 6 in Kelly et al. (1985) while Fig. 1b shows the regenerated data from Fig. 4 in Wakimoto (1985). Here, it can be seen that severe wind events occur most often during the late afternoon. As the sun begins to set, the number of severe wind events begins to sharply decrease. However, late evening and even nocturnal events, while less common, do occur for all wind events (Fig. 1a) and downbursts (Fig. 1b).

The period of the day roughly $2 \mathrm{~h}$ before sunset to $2 \mathrm{~h}$ after sunset is known as the evening transition (ET), when, in a matter of hours, the atmospheric boundary layer (ABL) transitions from the daytime convective boundary layer (CBL) to the nighttime stable boundary layer (SBL). The CBL is characterized by large turbulent eddies spanning the depth of the boundary layer generated from the heating of the surface. As the sun sets and the surface begins to cool, the SBL develops where a surface-based layer of stably stratified air forms along with an increase in low-level vertical wind shear. Fur- ther, due to the lack of surface heating, the turbulence from large convective eddies is greatly reduced (Stull, 1988). The neutral boundary layer (NBL) exists only very briefly between the convective and stable regimes (Park et al., 2014) where vertical sensible heat flux near the surface is zero and there is an adiabatic lapse rate throughout the boundary layer (Stull, 1988). At the beginning of the ET, when the boundary layer is still in a convective state, solar heating is able to generate convective instabilities in the boundary layer that can initiate thunderstorms (Wakimoto, 2001). However, as the sun sets, this convective instability source is lost, thus decreasing the number of such severe events.

Another interesting observation can be made from Fig. 1a: as the atmospheric stability increases during the ET, the percentage of events considered strong or violent remains relatively constant. In other words, atmospheric stability does not seem to play a role in modulating the ratio of severe to non-severe wind events. This counterintuitive result should be studied in detail. In fact, the impact of increased stability on tornadogenesis has been investigated recently; the climatologies of tornadic events have suggested a peak in the occurrence frequency of tornadoes in the early evening (Coffer and Parker, 2015). In the present study, we will probe further into how stability impacts downburst winds and into such implications on the wind energy and wind engineering communities. Other extreme wind events (derechos, rear flank downdrafts, etc.) are out of scope of the present study.

Downbursts, defined as such by Fujita (1985), produce low-level diverging outflow capable of generating severe winds. Such outflow is dominated primarily by the horizontal components of the wind field; however, they are enhanced by strong vertical motions at low levels as well. Downbursts are typically associated with thunderstorm activity and are commonly separated into two categories: dry and wet (Wakimoto, 1985). Dry downbursts are associated with little or no rain at the surface during the outflow winds. In contrast, wet downbursts are associated with rainfall at the surface during the time of the outflow winds. In order to understand distinctions between a downburst classified as dry or wet and the potential impact of atmospheric stability on downburst winds, basic concepts of downburst formation are explained here. The two main drivers to generate a downburst are hydrometeor loading (i.e., drag from falling hydrometeors) to initiate the sinking motion and latent cooling from melting, evaporation, and sublimation of the hydrometeors (Wakimoto, 2001). These produce a pocket of cold (relative to the environment), negatively buoyant air that descends towards the surface and spreads out laterally as it reaches the ground. With that basic picture in mind, several factors (e.g., the ambient turbulent motions of the CBL or the surface inversion with increased low-level wind shear in the SBL) play a role in modifying the downburst winds. For example, in a typical CBL or NBL regime, the pocket of air would remain negatively buoyant throughout its descent to the surface. However, in the SBL, where a low-level inversion is present, the 
downburst air could become less negatively buoyant (or even positively buoyant) as it descends through the low-level stable layer (Proctor, 1989). Since the resulting horizontal wind speeds are a function of the maximum downdraft velocity, $w_{\text {min }}$, if $w_{\text {min }}$ is smaller (larger) then the outflow wind speeds will in turn be smaller (larger) (Proctor, 1989; Mason et al., 2009). Thus, if the downdraft encounters a highly turbulent environment, the mixing and entrainment of ambient environmental air can weaken the negative buoyancy of the parcel and, in turn, decrease the outflow wind speeds.

For several decades, the wind engineering and the atmospheric science communities have studied downburst winds. Due to a lack of high-resolution low-level observations during these events, as previously mentioned, numerical models as well as laboratory experiments (e.g., Lundgren et al., 1992; Yao and Lundgren, 1996; Sengupta and Sarkar, 2008; Zhang et al., 2013; Jesson et al., 2015) have been utilized to study downburst dynamics and wind fields. Numerical models employed include analytical models, Reynolds-averaged Navier-Stokes (RANS) models, large eddy simulation (LES) models, and mesoscale meteorological models.

Initially, based on observations, analytical models were developed in order to assess the danger of downbursts to aircrafts (Bowles and Frost, 1987; Vicroy, 1991). Such studies were, in a large part, motivated by several commercial airline accidents (e.g., the Delta Airlines Flight 191 crash at DallasFort Worth in 1985 with 137 fatalities; Fujita, 1986) and an earlier high-profile near miss at Andrews Air Force Base in 1983 in which Air Force One carrying then President Reagan landed just $6 \mathrm{~min}$ before wind speeds had reached $67 \mathrm{~m} \mathrm{~s}^{-1}$ at $5 \mathrm{~m}$ above the surface (Fujita, 1985). Developments stemming from these early analytical models proved to be useful in wind engineering studies (Holmes and Oliver, 2000; Chay et al., 2006; Chen and Letchford, 2004) and to wind energy research (Nguyen et al., 2010, 2011, 2013; Nguyen and Manuel, 2013, 2015). These relatively simple models are very efficient and inexpensive to run at high resolution, making them the state of practice in the wind engineering community. However, these models fall short of representing the underlying physics accurately and, especially with regard to turbulence, they rely on synthetically generated stochastic fields associated with target power spectral density functions, which are not specifically associated with downbursts.

RANS simulations of downburst winds (Mason et al., 2009, 2010a; Proctor, 1988) and LES studies (Vermeire et al., 2011a; Anabor et al., 2011; Orf et al., 2012, 2014) both solve the Navier-Stokes equations and have been used to study the dynamics of downburst winds. What separates the two methods is that RANS models estimate the entire turbulent contributions of the flow field, while LES resolves the largest turbulent eddies and parameterizes only the smaller turbulent eddies. An advantage of both approaches is that several fundamental physical features of downburst flows can be simulated. For example, RANS studies performed by Proctor (1988) produced, to the best of our knowledge, the first simulation of what is known as the "ring vortex" - a distinctive feature commonly observed in downbursts (Fujita, 1985; Smith, 1986; Kessinger et al., 1988; Wakimoto, 2001). This feature is characterized by the rotation (roll-up) around a horizontal axis located towards the edge of the outflow and commonly associated with the strongest outflow winds. Further, the influence of various other factors such as terrain (Mason et al., 2010b), the interactions of colliding downburst outflows (Vermeire et al., 2011b), and the microphysics and ambient humidity (Proctor, 1989, 1988; Srivastava, 1985, 1987) have all been explored. Full-storm simulations utilizing advanced parameterizations (Orf et al., 2012, 2014) are now able to help us advance our knowledge of downburst dynamics and study these various influences. A major drawback of RANS, LES, and full-storm simulations is the computational expense involved in obtaining high-resolution spatiotemporal representations of downbursts. As a result, these models are rarely run at resolutions suitable for use as inflow in engineering models.

To the best of our knowledge, all previously published numerical studies of downburst winds have only considered downbursts in the NBL with one exception - a sensitivity study by Proctor (1989) that used a RANS model. In that study, surface-based deep inversion layers of 500 and $1000 \mathrm{~m}$ were used to mimic nocturnal stability or the outflow of another storm. It was shown that the stable layer at the surface reduced the magnitude of the outflow wind speed and the maximum downdraft; it also prevented one downburst event from even reaching the surface entirely. The height of the ring vortex that formed was also shown to be sensitive to the surface-based inversion in that this height was equivalent to (or the same as) that of the stable layer. This single study only describes part of the influence of the SBL; the expected characteristic increase in low-level wind shear, a fundamental feature of the SBL, was ignored.

When considering the three planetary boundary layer (PBL) regimes, it is to be expected that initial studies would only consider neutral conditions. The NBL provides the most simplistic ambient environment in that it lacks several complex processes inherent to the CBL and SBL. However, since thunderstorms and downbursts occur throughout the night and day, it is necessary to assess what effect stability might have on downburst outflows. In the present study, a pseudospectral LES model with a dynamic sub-grid-scale (SGS) scheme is utilized to simulate downbursts in various stability regimes. The goal of this study is to analyze outflow wind fields to learn how downburst intensity and flow characteristics change with increased low-level stability. Section 2 introduces the methodology employed in this study. Results for the ET and the downburst simulations are presented in Sect. 3.1 and 3.2, respectively. Finally, a summary and conclusions are provided in Sect. 4. 

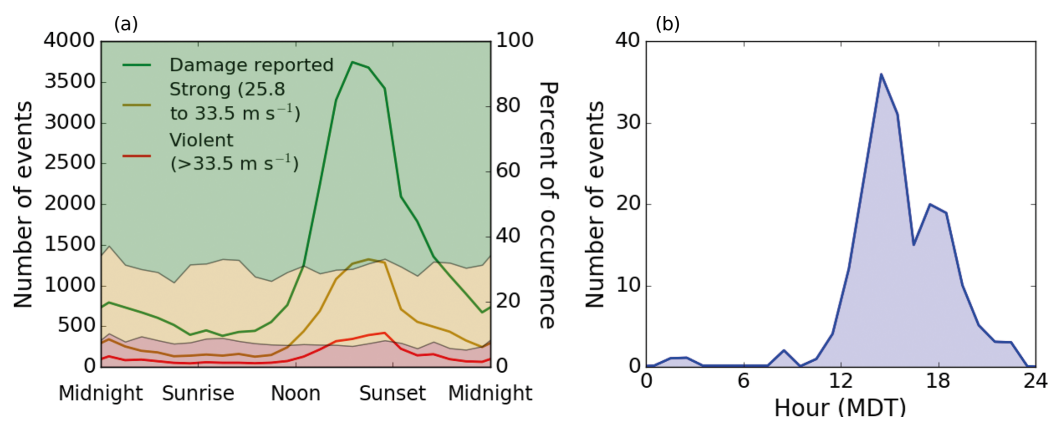

Figure 1. (a) The frequency of wind gusts of varying severity throughout the day (solid lines) overlaying the percentage of each wind gust severity category. Red, yellow, and green coloring correspond to violent gusts (greater than $33.5 \mathrm{~m} \mathrm{~s}^{-1}$ ), strong gusts (between 25.8 and $33.5 \mathrm{~m} \mathrm{~s}^{-1}$ ), and events with reported damage but no estimated wind speeds, respectively. Data have been adapted from Kelly et al. (1985), where the times have been converted to normalized solar time (NST). (b) Time of occurrence of the downbursts observed in the JAWS campaign; regenerated data from Fig. 4 in Wakimoto (1985).

\section{Methods}

Simulating the diurnal cycle has been a challenging topic in boundary layer meteorology for some time. In convective simulations, the model domain size must be large enough in both the horizontal and vertical extent to capture the convective processes. Due to these large domains, modelers are typically constrained to using relatively coarse grid sizes to decrease the computational burden of each simulation. However, when simulating the SBL, the model grid spacing and time step must be small enough to capture developing nighttime eddies that are on the order of meters. In order to satisfactorily simulate both regimes, a large model domain with small grid spacing is necessary; this results in the need for a large amount of computational grids. With advances in computational power, we have began to be able to run these types of simulations with relative ease.

Basu et al. (2008) used a dynamic SGS scheme, called the locally averaged scale-dependent dynamic (LASDD) model (Basu and Porté-Agel, 2006), to simulate a full diurnal cycle. The results showed good agreement with observations and, additionally, little dependence on model grid spacing. In the LASDD SGS model, the SGS coefficients are computed dynamically based on the local dynamics of the flow, whereas in typical SGS schemes the SGS coefficients are specified in an ad hoc manner.

The LES model used in Basu et al. (2008) is utilized here to simulate the idealized downbursts. The model forcing is imposed by specifying the geostrophic wind and the surface temperature. The model domain spans $10 \mathrm{~km}$ in both lateral directions and $3 \mathrm{~km}$ in the vertical. The grid spacing is approximately $28 \mathrm{~m}$ in both the horizontal and vertical directions. The model's lateral boundaries are periodic in both the $x$ and $y$ direction, and a damping layer is employed on the top $1 \mathrm{~km}$ of the domain in order to eliminate reflections off of the model top. A roughness length of $0.1 \mathrm{~m}$ is utilized corresponding closely to the average roughness length over North
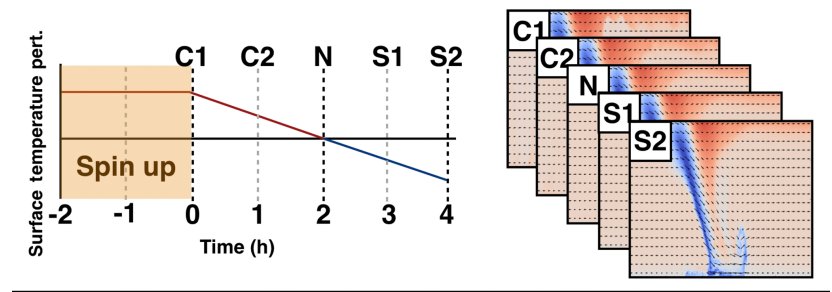

Phase I

Phase II

Phase III

Figure 2. A schematic diagram depicting the process for simulating downbursts throughout the evening transition period. The first phase consists of model spin-up to generate the CBL. The second phase simulates the ET while outputting a snapshot every hour to be used as the initial and boundary conditions in the downburst simulations performed in Phase III. These snapshots are denoted as $\mathrm{C} 1$ for the moderately convective case, $\mathrm{C} 2$ for the weakly convective case, $\mathrm{N}$ for the neutral case, S1 for the weakly stable case, and S2 for the moderately stable case.

America (Stull, 1988). The simulations of downbursts during the evening transition is conducted in three phases (Fig. 2).

\subsection{Phase I: spin-up simulation}

The model is initialized with a neutral potential temperature profile up to the initial boundary layer height of $1.5 \mathrm{~km}$ and a constant geostrophic wind of $8 \mathrm{~m} \mathrm{~s}^{-1}$ in the positive- $x$ direction (i.e., $U_{\mathrm{g}}=8.0 \mathrm{~m} \mathrm{~s}^{-1} ; V_{\mathrm{g}}=0.0 \mathrm{~m} \mathrm{~s}^{-1}$ ). A prescribed surface temperature is used as the lower boundary condition to drive the flow. In this case, the initial temperature in the NBL is $300 \mathrm{~K}$ and the surface temperature is prescribed to be $303 \mathrm{~K}$. For the first $2 \mathrm{~h}$ of the simulation, the constant surface temperature is used to develop the convective boundary layer. The model time step, $\Delta_{t}$, is set to $0.5 \mathrm{~s}$. These $2 \mathrm{~h}$ are regarded as the period of model spin-up and data from this phase are not used in subsequent analysis. 


\subsection{Phase II: ET simulation}

In the second phase, the ET is simulated for $4 \mathrm{~h}$, during which the surface temperature is decreased by $1.135 \mathrm{~K} \mathrm{~h}^{-1}$ such that after $2 \mathrm{~h}$ the boundary layer will reach a neutral state and the SBL will be developed in the subsequent $2 \mathrm{~h}$. The selected surface temperature cooling rate was found by trial and error in order to produce a neutral state after $2 \mathrm{~h}$ of cooling. In this phase, the same $\Delta_{t}$ of $0.5 \mathrm{~s}$ is used as in Phase I and, after every hour of simulation, the full three-dimensional velocity and temperature fields are output to be used as initial and boundary conditions for Phase III simulations. These snapshots are denoted as $\mathrm{C} 1$ for the moderately convective case, $\mathrm{C} 2$ for the weakly convective case, $\mathrm{N}$ for the neutral case, $\mathrm{S} 1$ for the weakly stable case, and S2 for the moderately stable case; they are output at hours $0,1,2,3$, and 4 , respectively.

\subsection{Phase III: downburst simulations}

The downburst simulations are initialized from the $\mathrm{C} 1, \mathrm{C} 2$, $\mathrm{N}, \mathrm{S} 1$, and S2 snapshots of the ET simulation. In these simulations, $\Delta_{t}$ is reduced to $0.1 \mathrm{~s}$ and each simulation is run for $15 \mathrm{~min}$. This decrease in time step is made to ensure stability and for the collection of high-temporal-resolution data to be used in a follow-up study utilizing the Fatigue, Aerodynamics, Structures, and Turbulence (FAST) model. Due to the sensitivity of LES runs to initial conditions, an ensemble of simulations is generated for each stability case $(\mathrm{C} 1, \mathrm{C} 2, \mathrm{~N}$, $\mathrm{S} 1$, and S2). Each case is run four times; taking advantage of the periodic boundaries in the $x$ - and $y$ directions, the initial conditions generated from the ET simulation are shifted as follows: (1) in the $x$ direction by $+5 \mathrm{~km}$ (denoted by 5x_0y), (2) in the $y$ direction by $+5 \mathrm{~km}$ (denoted by $0 x \_5 y$ ), and (3) in both the $x$ and $y$ directions by $+5 \mathrm{~km}$ (denoted by $5 \mathrm{x} \_5 y$ ). Including the original initial conditions, this strategy results in four sets of initial conditions for each case. Shifting the initial conditions in this manner effectively initializes the downburst at different locations within the same ABL regime.

\subsection{Downburst forcing}

A cooling source near the top of the model originally proposed by Anderson et al. (1992) is used to produce negatively buoyant air to simulate the effects of latent cooling due to the melting, evaporation, and sublimation of hydrometeors as they descend towards the surface. These processes are largely responsible for generating the negative buoyancy that drives the downburst (Wakimoto, 2001) and this approach has become the state of the practice in LES simulations of downburst winds (see, for example, Orf et al., 1996; Anabor et al., 2011, or Oreskovic, 2016). The source ( $Q$ ) equations are as follows:

$$
Q(x, y, z ; t)=\left\{\begin{array}{ll}
C_{\max } \times g(t) \cos ^{2}(\pi R), & R \leq \frac{1}{2} \\
0, & R>\frac{1}{2}
\end{array},\right.
$$

where $C_{\max }$ is the peak intensity of the cooling function and is set to $-0.08 \mathrm{~K} \mathrm{~s}^{-1}$ as was done in Mason et al. (2009) and Anabor et al. (2011) to produce an intense downburst. The function, $g(t)$, defines the variation of downburst intensity with time as follows:

$g(t)= \begin{cases}\cos ^{2}\left[\pi\left(\frac{t-120}{2 \tau}\right)\right], & 0<t \leq 120 \\ 1, & 120<t \leq 240 \\ \cos ^{2}\left[\pi\left(\frac{t-240}{2 \tau}\right)\right], & 240<t \leq 360\end{cases}$

where $\tau$ is set to $120 \mathrm{~s}$. Equation (2) differs from previous studies in that the cooling source is held constant for $2 \mathrm{~min}$, as opposed to $10 \mathrm{~min}$. This is done in order to analyze the decay of the downburst events before they reach the simulation boundary as well as to limit the source from generating multiple strong downburst events. Finally, $R$ represents the normalized distance from the center of the cooling function (Vermeire et al., 2011a; Anabor et al., 2011):

$R=\sqrt{\left(\frac{x-x_{f}}{M_{x}}\right)^{2}+\left(\frac{y-y_{f}}{M_{y}}\right)^{2}+\left(\frac{z-z_{f}}{M_{z}}\right)^{2}}$.

Here, the coordinates of the center of the downburst, $\left(x_{f}, y_{f}, z_{f}\right)$, are set to $(2.5,5.0,1.9 \mathrm{~km})$. The $x$ position is chosen so as to allow the downburst to reach the surface near the center of the domain and extend the amount of time before the downburst reaches the model boundary. The vertical location of the cooling center is chosen such that it is concentrated just inside the entrainment zone overlying the mixed layer. The horizontal and vertical extent of the cooling source, defined by $M_{x}, M_{y}$, and $M_{z}$, are set to 2.0, 2.0, and $1.5 \mathrm{~km}$, respectively, such that the vertical extent of the cooling source does not reach the surface. This type of cooling source essentially mimics latent cooling from a dry microburst in which most, if not all, of the hydrometeors melt, evaporate, and/or sublimate before reaching the ground. The cooling source is stationary as is common in the downburst literature (Anderson et al., 1992; Mason et al., 2009, 2010a; Anabor et al., 2011; Vermeire et al., 2011a, b; Oreskovic, 2016). Mason et al. (2010a) compare this method with a translating source and conclude that both are viable methods for downburst simulations.

\section{Results}

A brief analysis of the evening transition simulation (which provides the initial conditions for the downburst simulations) is presented in Sect. 3.1, followed by a detailed analysis of the downburst cases in Sect. 3.2. The analysis performed herein is from the lowest $2 \mathrm{~km}$ of the simulation domain in order to limit any influence from the damping layer.

\subsection{ET simulation}

The evening transition simulation produces hourly restart files to be used as the initial conditions for each of the down- 

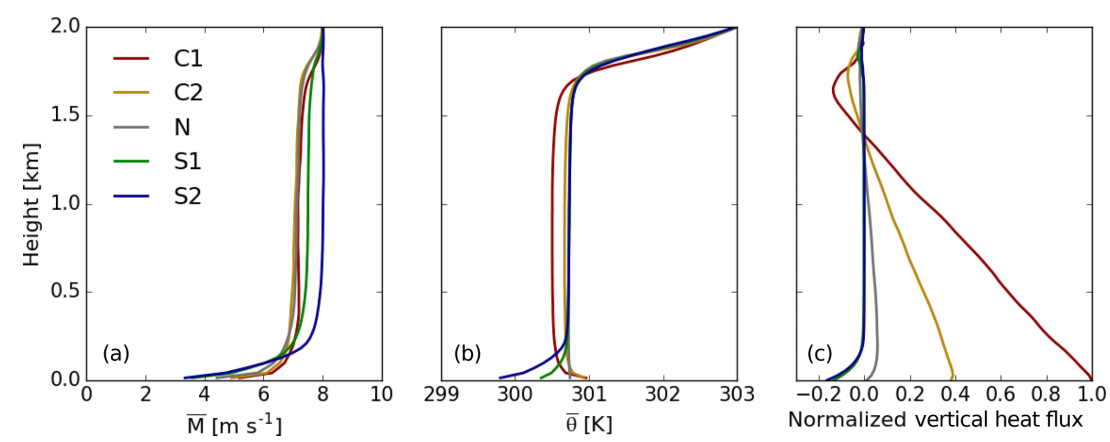

Figure 3. Vertical profiles of average wind speed (a), temperature (b), and normalized vertical sensible heat flux (c) are shown for the C1, C2, $\mathrm{N}, \mathrm{S} 1$, and S2 cases colored red, yellow, gray, green, and blue, respectively. The normalized vertical sensible heat flux profiles are normalized with respect to the average surface sensible heat flux of the $\mathrm{C} 1$ simulation.

burst simulations. The potential temperature and wind velocity profiles for each case are shown in Fig. 3. As can be seen, both the $\mathrm{C} 1$ and $\mathrm{C} 2$ temperature profiles (red and yellow lines, respectively) are unconditionally unstable throughout the boundary layer with the instability reducing to zero by case $\mathrm{N}$ (gray line). Even though the surface temperature is decreasing between the $\mathrm{C} 1$ and $\mathrm{C} 2$ cases, the prescribed temperature is still larger than that of the mixed layer. The warmer surface combined with the warming from entrainment (mixing of the warm air above the boundary layer into the mixed layer) results in a continual warming in the mixed layer between $\mathrm{C} 1$ and $\mathrm{C} 2$. As the simulation progresses, a surface-based stable layer develops by $\mathrm{S} 1$ and deepens to $\mathrm{S} 2$ (green and blue lines, respectively). The mixed layer temperature between the $\mathrm{C} 2$ and $\mathrm{N}$ cases continues to subtly warm. In previous ET studies, it has been shown that entrainment can persist due to residual convection after the surface heating has stopped (Nieuwstadt and Brost, 1986; Sorbjan, 1997). During the generation of the stable layer, the average wind speed throughout the residual layer (remnant of the mixed layer) increases along with the deepening of shear below roughly $250 \mathrm{~m}$. This low-level stable layer is estimated to be as deep as $200 \mathrm{~m}$.

The vertical sensible heat flux profiles are normalized relative to the surface sensible heat flux of the $\mathrm{C} 1$ case. It can be seen here that over time the amount of vertical sensible heat flux near the surface and within the mixed layer decreases to zero by the $\mathrm{N}$ case and becoming negative after. The decrease of vertical sensible heat flux throughout the boundary layer occurs from the bottom up as is consistent with the literature (Nieuwstadt and Brost, 1986). The ET is defined as the period in which the boundary layer becomes approximately neutral near the surface and achieves a vertical sensible heat flux of zero at the lowest model level. The negative portion of vertical sensible heat flux above roughly $1.5 \mathrm{~km}$ is caused by entrainment. The height at which the vertical sensible heat flux is most negative is often used to define the daytime boundary layer height. Entrainment acts to increase the boundary layer height most notably between $\mathrm{C} 1$ and $\mathrm{C} 2$.
Figure 4 shows a vertical cross section of the $w$ component velocity field for the C1-5x_5y (top row), N$5 \mathrm{x} \_0 \mathrm{y}$ (middle row), and S2-0x_5y (bottom row) cases along with average profiles of the $u$-and $v$-component winds in the right column. These cross sections are taken from the location of maximum vertical velocity in order to get a sense for the strongest of the updrafts within the domain. As can be seen in the $\mathrm{C} 1-5 \mathrm{x} \_5 \mathrm{y}$ simulation, strong thermals are present within the model domain in which vertical velocities reach over $6 \mathrm{~m} \mathrm{~s}^{-1}$. As the surface heating decreases, the strength of the thermals becomes weaker. However, by the time the atmosphere reaches a near-neutral regime, remnants of these thermals are still prevalent throughout the domain. After 2 more hours of stabilization, these remnants have largely dissipated as shown in the S2-0x_5y panel.

Analyzing the average profiles of the $u$ - and $v$-component winds in Fig. 4, it is clear to see the increase in $v$-component wind speeds as stability increases. In the stable cases, a sharp increase in the $v$-component winds below roughly $100 \mathrm{~m}$ is seen due to the Coriolis force causing the winds to veer with height within the SBL (Stull, 1988).

\subsection{Downburst simulations}

The downburst simulations are each run for $15 \mathrm{~min}$. After about $13 \mathrm{~min}$, the outflow reaches the domain boundary in each simulation. For this reason, only data from the vertical plane at $x=6.0 \mathrm{~km}$ are analyzed for the full $15 \mathrm{~min}$ as the boundaries do not influence these results. Between the different downburst simulations, only the initial and boundary conditions are changed. Surface temperature is decreased, as within the ET simulation, for the full downburst duration. For generalization purposes, the ensemble mean for several of the analysis fields is presented here. However, in order to gain insight into the individual downbursts, a characteristic simulation is chosen from each case for certain analyses. Downburst simulation results are presented by referring to time in

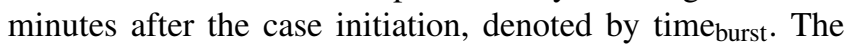




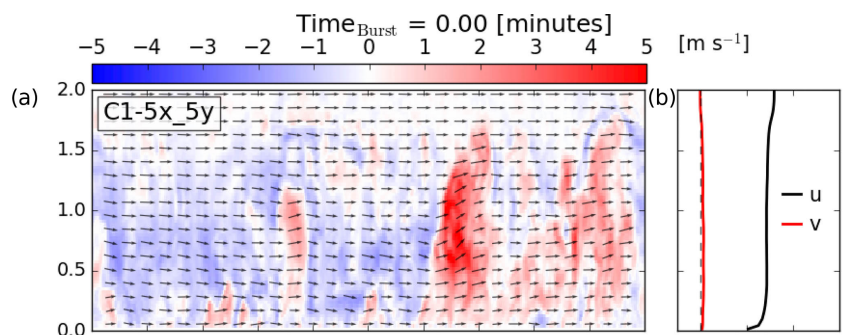

(c)
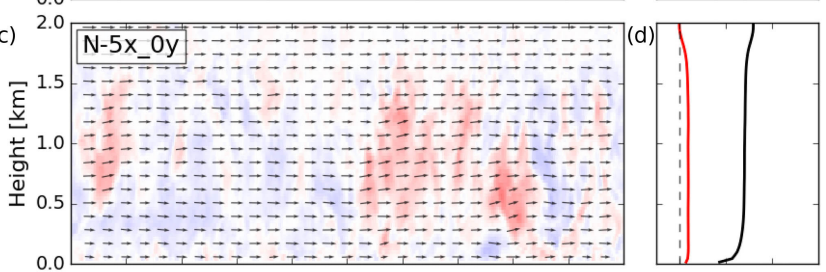

(e)

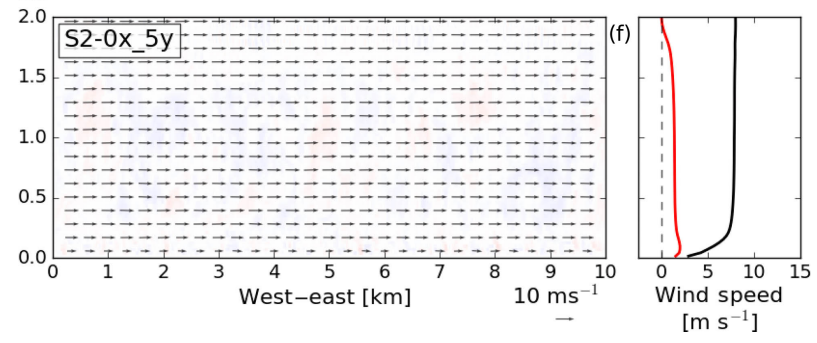

Figure 4. Vertical cross sections of vertical velocity and average profiles of the $u$ - and $v$-component winds for the $\mathrm{C} 1-5 \mathrm{x} \_5 \mathrm{y}(\mathbf{a}, \mathbf{b})$, N-5x_0y (c, d), and S2-0x_5y (e, f) cases at the start of the downburst runs. Cross sections are taken along the east-west axis at the $y$ location where the maximum vertical velocity occurs. Filled contours show vertical velocity $\left(\mathrm{m} \mathrm{s}^{-1}\right)$ with arrows representing the $u-w$ wind field. The domain average $u$ - and $v$-component wind speed profiles are shown in the right column in black and red, respectively.

hours into the ET simulation at which the downburst cases are initiated can be seen in the schematic presented in Fig. 2.

\subsubsection{Velocity fields}

In each of the downburst simulations, a diverging outflow pattern in the horizontal wind fields is generated as shown in Fig. 5. The initial (top row) and mature-to-dissipating (bottom row) stages of the downbursts are shown for the C1-5x_5y (left column), N-5x_0y (center column), and S20x_5y (right column) cases. These horizontal cross sections are taken at around $100 \mathrm{~m}$ above ground level, a typical value for the hub height of a commercial wind turbine. The initial stages of the downbursts show large swaths of areas with horizontal winds greater than $20 \mathrm{~m} \mathrm{~s}^{-1}$ (denoted by black arrows), mostly confined to regions downwind of the downburst (i.e., to the right of the downburst center). At this stage, the $\mathrm{C} 1$ and $\mathrm{N}$ cases appear to generate generally stronger horizontal winds than the stable, S2, case. However, below $100 \mathrm{~m}$, the wind speeds for all cases increase (not shown), suggesting that the wind speeds in the S2 case are simply confined to lower heights than the other cases. As the outflow evolves, by time burst $=10.25 \mathrm{~min}$, the $\mathrm{S} 2$ case has fully developed a large, strong horizontal outflow region while the convective case has begun to dissipate. It can be seen that the ambient winds in the $\mathrm{C} 1$ case have much more variability than the $\mathrm{N}$ and $\mathrm{S} 2$ cases, which effectively deforms and disorganizes the outflow.

The large regions of high winds extending generally in the north-south direction in Fig. 5 are organized by the ring vortex. This feature can be seen near the vertical dashed line in Fig. 6 for each of the presented cases. The ring vortex increases the wind speed beneath it; the center of this ring vortex occurs at increasingly higher elevations as stability increases. The depth of the head of the outflow is also seen to increase with increasing stability due to the interactions of the ambient winds with the outflow - a similar result to what has been reported based on RKW theory (see Rotunno et al., 1988, for details). This theory shows how low-level shear can interact with outflow to generate stronger, more vertically upright updrafts from the counteracting vortices from the outflow and the ambient environment. Interestingly, a similar result is also reported in the simulations by Proctor (1989), where, by only introducing a surface-based temperature inversion, the depth of the outflow and the height of the ring vortex increase to equal the depth of the stable layer. In the present environment, both an increase in low-level shear and a surface-based temperature inversion are considered and a qualitatively similar trend is observed.

The vertical wind shear generated from the development of the ring vortex can easily be seen in the vertical profiles of horizontal (black) and vertical (blue) winds in Fig. 6 (right panels). These profiles are taken at the center of the domain in the $y$ direction and at the location of the maximum wind speed in the $x$ direction. In each case, the maximum wind speed occurs behind the center of the ring vortex. The associated upward motions (see the S2-0x_5y panel) and downward motions (see the $\mathrm{C} 1-5 \mathrm{x} \_5 \mathrm{y}$ and $\mathrm{N}-5 \mathrm{x} \_0 \mathrm{y}$ panels) can be seen in the vertical velocity profile. These motions are confined mostly to the lowest $500 \mathrm{~m}$. The vertical structure in the horizontal winds in case $\mathrm{C} 1-5 \mathrm{x} \_5 \mathrm{y}$ shows an increasing horizontal wind from the height of the ring vortex down to the surface. Cases N-5x_0y and S2-0x_5y, however, show a vertical structure below around $250 \mathrm{~m}$ that is quite complex. The wind speeds decrease from the lowest level to around $150 \mathrm{~m}$, then sharply increase again before quickly decreasing to ambient wind speeds at $500 \mathrm{~m}$. The strongest horizontal wind speeds occur at the lowest model level in these cases, but the secondary peak above this appears to be associated with the temperature gradient generated by the warm air entraining into the ring vortex. It should be noted that these complex wind patterns are occurring at heights that would impact typical commercial wind turbines.

To get an idea of the temporal structure at a single point during the passage of the downburst, Fig. 7 shows the magnitude of velocity ( $M$; black line) and the individual compo- 

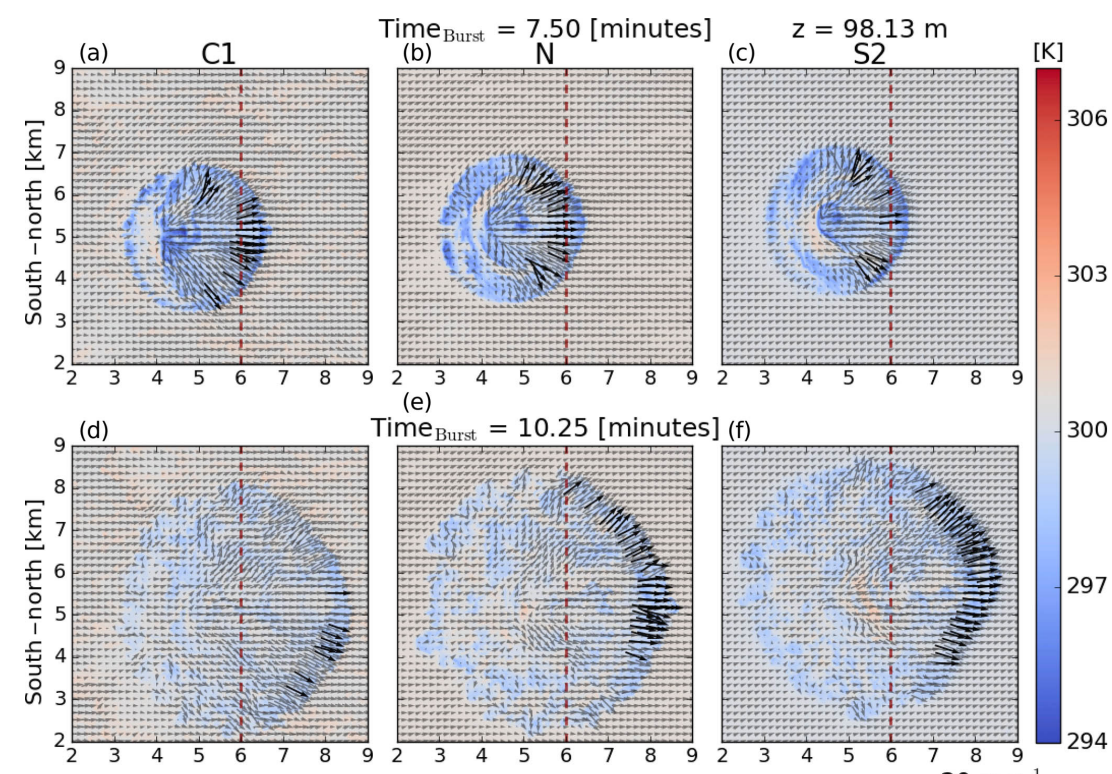

Time $_{\text {Burst }}=10.25$ [minutes $](f)$

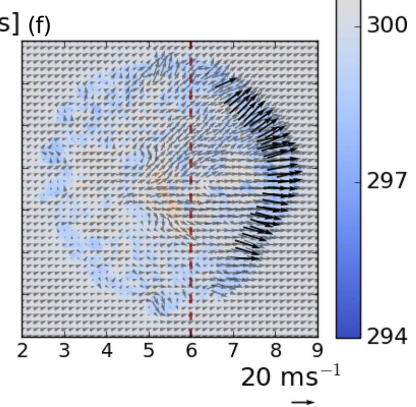

Figure 5. Horizontal cross sections at $98.13 \mathrm{~m}$ for the C1-5x_5y (a, d), N-5x_0y (b, e), and S2-0x_5y (c, f) cases at time burst $=7.5 \mathrm{~min}(\mathbf{a}$, b, c) and $10.25 \mathrm{~min}(\mathbf{d}, \mathbf{e}, \mathbf{f})$. Filled contours show the temperature $(\mathrm{K})$ with arrows representing the horizontal wind field at this level. The locations with wind speeds above $20 \mathrm{~m} \mathrm{~s}^{-1}$ are shaded in black, while locations with wind speeds below this threshold are shaded in gray. The north-south-oriented line denotes the location at which a vertical slice is recorded at every time step used to generate the time series in Fig. 7.

nents of the wind field $(u, v$, and $w)$ in red, green, and blue, respectively, at $y_{\max }$. Here, $y_{\max }$ is defined as the location along the vertical cross sections at which the maximum horizontal wind speed occurs at $98.13 \mathrm{~m}$ above the ground over the full 15 min duration. This location will change from case to case as the maximum wind speeds along this cross section do not always happen at the same location. The time series are shown at $98.13 \mathrm{~m}$ for direct comparison with Fig. 5 for each of the simulations in cases $\mathrm{C} 1$ (panel a), $\mathrm{N}$ (panel b), and S2 (panel c). Each of the ensemble members are shown to highlight the extent of the deviation between runs. By studying the vertical velocity time series (blue line) for each case, the time between peak-positive and peak-negative vertical wind speeds (indicative of the ring vortex passage) is seen to be as short as $20 \mathrm{~s}$ to as long as about $1 \mathrm{~min}$. Generally, this time between these positive and negative peak values increases as stability increases. This is due to the associated increased spatial extent of the ring vortex or due to a decrease in the speed at which it propagates (Proctor, 1989, found that a low-level temperature inversion decreased the propagation speed of the outflow). Each case also shows the maximum wind speed occurring in between the maximum updraft and maximum downdraft, consistent with the passage of the ring vortex.

In the $\mathrm{C} 1$ simulations, the vertical velocity and lateral velocity components are quite low relative to the other simulations. This is because $y_{\max }$ is located closer to the center of the domain and because a weaker ring vortex forms in these convective simulations. In the neutral simulations, the $y_{\max }$ locations are split between north of center and south of center (indicated by the positive and negative peaks, respectively, in the $v$-component wind speeds), hinting at a lack in preferential location of occurrence of the maximum wind speed. In contrast, in the $\mathrm{S} 2$ simulations, all of the $y_{\max }$ locations are north of center, where the outflow propagation direction is normal to the direction of the low-level wind shear vector. A similar result was found in a numerical case study by Proctor (1994) of a dry, pulsating microburst event. In the current model, the low-level wind shear is generated by the Coriolis effect on the low-level stable layer (Stull, 1988). This is consistent with the added ambient shear in the stable cases that bolsters the ring vortex and, thus, helps to increase horizontal wind speeds at this location. Here, we also see that the peak horizontal wind speed reaches the vertical cross section at $x=6.0 \mathrm{~km}$ earlier in the $\mathrm{C} 1$ simulations and later in the S2 simulations. Although there are differences in the propagation speed from case to case, the differences in timing here are due to the circular nature of the downburst resulting in the ramp reaching locations along the vertical slice at different times. The location of $y_{\max }$ changes in each case, with the S2 cases showing a preference to locations along the slice further from the center of the domain, which results in the wind ramp occurring at a later time than the $\mathrm{C} 1$ and $\mathrm{N}$ cases. Lastly, the additional stability in the $\mathrm{S} 2$ cases allows the wind field to recover to pre-outflow values more rapidly; 


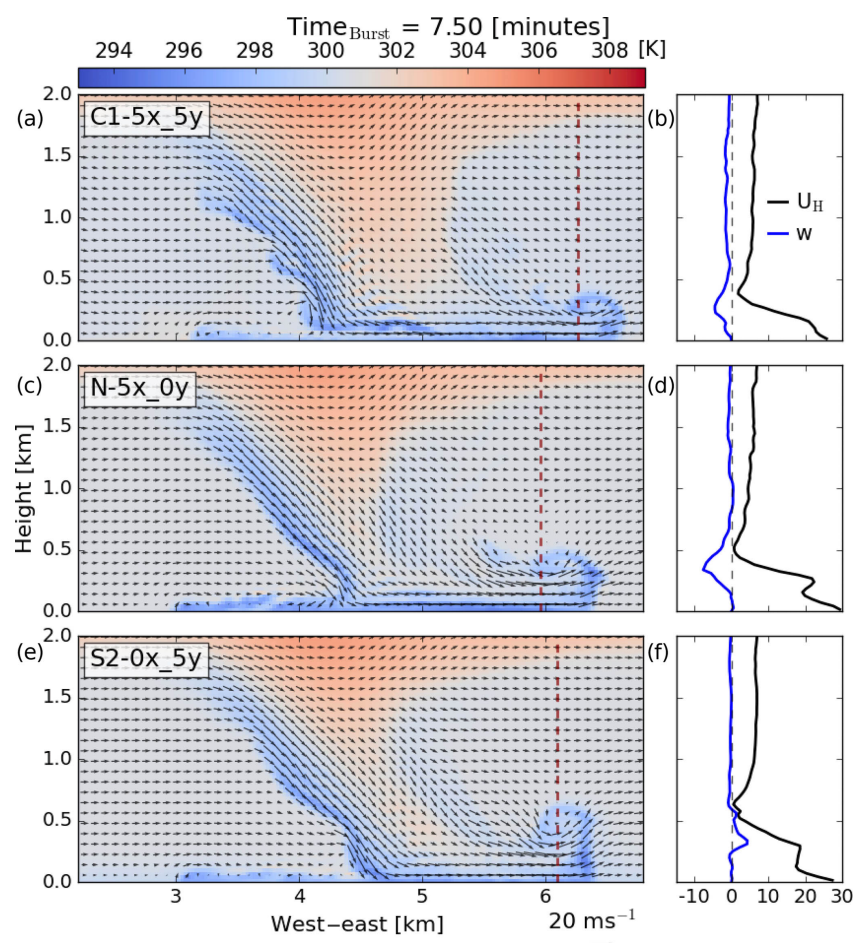

Figure 6. Vertical cross sections along the east-west axis at the center of the domain for the C1-5x_5y (a, b), N-5x_0y (c, d), and $\mathrm{S} 2-0 \mathrm{x} \_5 \mathrm{y}(\mathbf{e}, \mathbf{f})$ cases at time $\mathrm{burst}=7.5 \mathrm{~min}$. Filled contours show temperature $(\mathrm{K})$ with arrows representing the $u-w$ wind field. In panels $(\mathbf{b}, \mathbf{d}, \mathbf{f})$ are the horizontal velocity and vertical velocity profiles (black and blue, respectively) for each case at the dashed line in the vertical cross section (of $\mathbf{a}, \mathbf{c}, \mathbf{e}$ ).

this recovery is thus a function of the stability as well as the location of $y_{\max }$.

The ensemble mean of the domain-maximum horizontal wind speeds at each height with time is presented using filled contours in Fig. 8. Open contours of the ensemble mean of the maximum downdraft (black) and maximum updraft (dotted magenta) are also included in this figure in order to deduce the state of the downburst. Several generalizations can be made about the structure of the maximum wind speeds for each of the simulations. First, the initial signal of the downburst comes from a rapid increase in intensity of the downdraft. Then, horizontal wind speeds near the surface begin to increase; this is followed by a strengthening of the updraft wind speeds between around 100 and $300 \mathrm{~m}$. The peak wind speed is generally reached just after the updraft band develops with the exception of the $\mathrm{C} 1$ case, where the two appear to occur simultaneously in the ensemble-averaged output. Finally, as the updraft band begins to vertically expand, the horizontal outflow decreases. This happens at different times and rates for each case. The collocation of the updraft and downdraft bands is due to the ring vortex. When these bands expand and taper off, it is indicative of a ring vortex that has become disorganized and begun to dissipate.

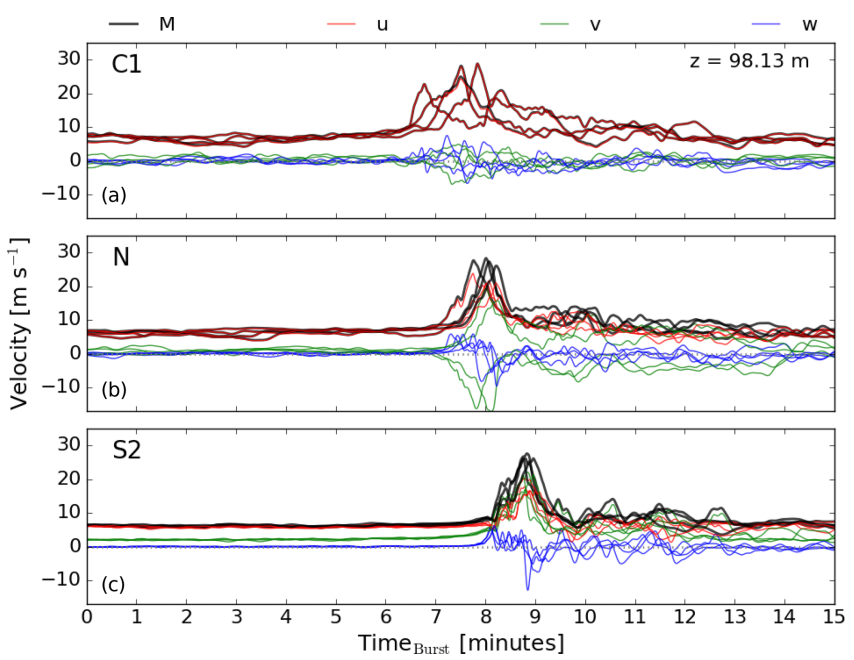

Figure 7. Time series of the velocity components at the location $\left(x=6 \mathrm{~km}, y=y_{\max }, z=98.13 \mathrm{~m}\right)$ for the $\mathrm{C} 1(\mathbf{a}), \mathrm{N}(\mathbf{b})$, and $\mathrm{S} 2(\mathbf{c})$ cases at the $6.0 \mathrm{~km}$ cross section (red dashed line in Fig. 5).

Inspecting $\mathrm{C} 1$, it is clear that the strength of the downdraft is weaker than the other simulations. This is due to the interaction of convective thermals and increased turbulent motions as the downburst descends through the column (see the top-left panel in Fig. 4). The impact of this interaction can be seen in Fig. 6, where the downdraft (between roughly 3.0 and $4.5 \mathrm{~km}$ in the west-east direction) is noticeably more disorganized in the $\mathrm{C} 1$ case but much more coherent in the $\mathrm{N}$ and $\mathrm{S} 2$ cases. By time burst $=11 \mathrm{~min}$ in Fig. 8, the downdrafts are no longer below $-10 \mathrm{~m} \mathrm{~s}^{-1}$ and the updrafts begin to ascend farther from the surface. This shows the demise of the ring vortex and coincides with the rapid weakening of the nearsurface winds.

As the stability increases, it can be seen that the maximum downdraft increases and that it also develops at a faster rate. Further, the initial depth of the strong horizontal winds produced from the outflow is contained closer to the surface with increasing stability, indicating an increase in outflowgenerated shear across the low levels of the atmosphere. Decay of the strong updrafts and downdrafts occurs at later times as stability increases, with case S2 showing the first signs of decay at time burst $=12 \mathrm{~min}$.

Several downburst modeling studies have attempted to find a relationship between the maximum outflow wind speed $\left(U_{\text {storm }}\right)$ to maximum downdraft $\left(w_{\min }\right)$ by calculating the ratio of $U_{\text {storm }}$ to $w_{\min }$ (Proctor, 1989, 1988; Mason et al., 2009; Anabor et al., 2011). Proctor (1989) showed that it was difficult to deduce any universal relationship between $U_{\text {storm }}$ and $w_{\min }$ in the brief study on the low-level stability. However, in his model, microphysics were being utilized and, thus, many more variables came into play in this scenario which makes deducing any relationships more complex. In this study, we attempt to determine relationships between this ratio and stability. Due to the peak wind speeds occurring so 


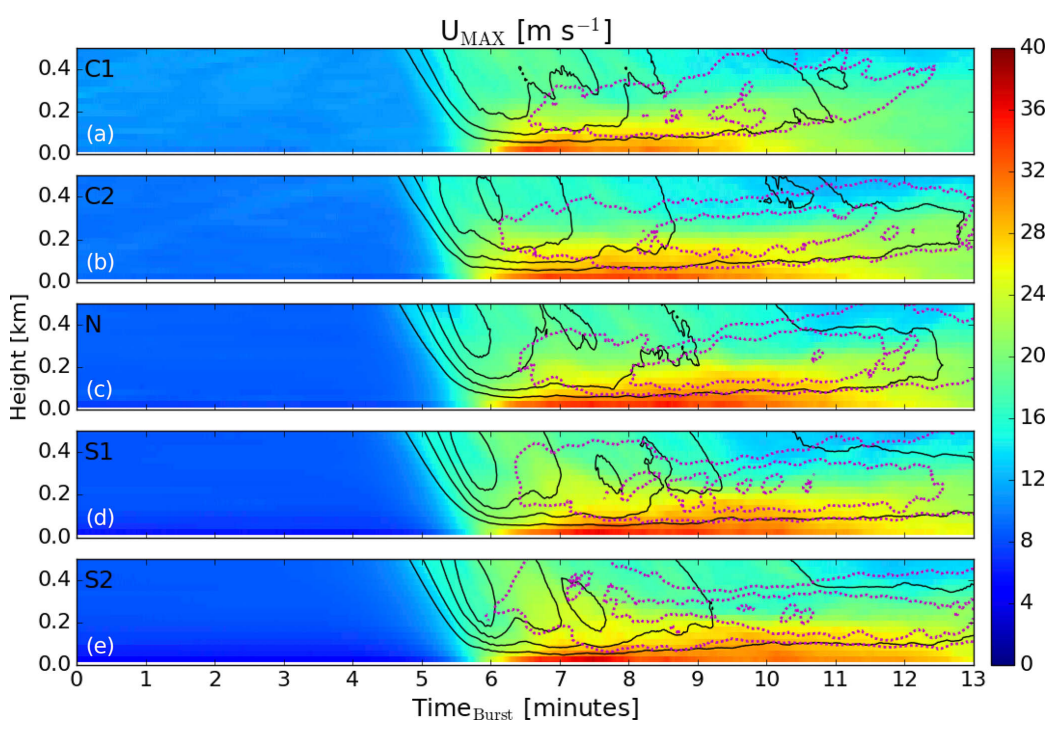

Figure 8. A time-height plot in the lowest $500 \mathrm{~m}$ of the ensemble average of maximum horizontal velocity for the $\mathrm{C} 1, \mathrm{C} 2, \mathrm{~N}, \mathrm{~S} 1$, and $\mathrm{S} 2$ cases going from panels (a-e). Contoured (in black) are the ensemble averages of minimum vertical velocity starting from $-10 \mathrm{~m} \mathrm{~s}^{-1}$ and drawn at $-5 \mathrm{~m} \mathrm{~s}^{-1}$ intervals and (in dotted magenta) the ensemble averages of maximum vertical velocity starting from positive $10 \mathrm{~m} \mathrm{~s}{ }^{-1}$ and drawn at $2.5 \mathrm{~m} \mathrm{~s}^{-1}$ intervals.

close to the surface, the vertical component is almost negligible; thus, in our computation of $U_{\text {storm }}$ we do not include the vertical velocity component (Mason et al., 2009).

Figure 9 shows plots of the $U_{\text {storm }}, w_{\min }$, and the ratio of $U_{\text {storm }}$ to $w_{\min }$ in the top, middle, and bottom rows, respectively, for the different simulations: $\mathrm{C} 1$ (red), $\mathrm{C} 2$ (yellow), $\mathrm{N}$ (gray), S1 (green), and S2 (blue). These quantities are plotted against the bulk Richardson number (Stull, 1988) at the lowest model level and averaged over the first 3 min of the simulation to deduce their relationships with stability. In studying the maximum outflow wind speed, a subtle and almost insignificant increase can be seen with increasing stability. The spread of the values tends to decrease as stability increases as well, owing to the heterogeneity of the convective simulations transitioning to the more homogeneous, stable regime. When considering only the ensemble mean values, as shown in Table 1, an increase with stability can be generally seen, although it is not an entirely monotonic increase. The maximum wind speeds of $35-38 \mathrm{~m} \mathrm{~s}^{-1}$ are consistent with strong observed downbursts: $25-30 \mathrm{~m} \mathrm{~s}^{-1}$ in Wakimoto (1985), $32 \mathrm{~m} \mathrm{~s}^{-1}$ (with theorized maxima in the $\mathrm{F} 3$ range of $70-92 \mathrm{~m} \mathrm{~s}^{-1}$ ) in Fujita (1981), and $67 \mathrm{~m} \mathrm{~s}^{-1}$ in Fujita (1985). Further, other observed modeling studies have generated similar wind speed maxima of $38 \mathrm{~m} \mathrm{~s}^{-1}$ (Orf et al., 2012), $35 \mathrm{~m} \mathrm{~s}^{-1}$ (Anabor et al., 2011), $57 \mathrm{~m} \mathrm{~s}^{-1}$ (Mason et al., 2009), 35-65 m s${ }^{-1}$ (Oreskovic, 2016), 24-32 $\mathrm{m} \mathrm{s}^{-1}$ (Orf and Anderson, 1999), and $47 \mathrm{~m} \mathrm{~s}^{-1}$ (Vermeire et al., 2011a).

In contrast, $w_{\min }$ is clearly strengthened with increasing stability as can be seen in Fig. 9 and Table 1. As previously mentioned, this is most likely attributed to the decrease in turbulence which would act to impede the downburst as it de- scends. In tracking the height at which the maximum downdraft occurs for each ensemble member, Table 1 shows a somewhat unclear relationship between the ensemble mean maximum downdraft and ensemble mean of the heights at which this downdraft occurs. Here, we can see that the higher downdraft wind speeds are occurring at lower heights. Intuitively, one might expect the stable surface layer to impede the progress of the downburst at lower heights and force the peaks to occur at higher levels. The opposite is seen in the cases herein, likely because the stable layer developed in the ET simulation is too shallow to impede the downdraft.

Finally, comparing the ratio of $U_{\text {storm }}$ to the magnitude of $w_{\min }$ in Fig. 9, we notice what appears to be a converging pattern to a value of roughly 1.3 as stability increases. The spread of values decreases significantly from the $\mathrm{C} 1$ cases to the two stable cases. Overall, the range of values of this ratio is in line with what other downburst simulations have produced (Proctor $(1988,1989)$ showed values ranging from 0.8 to 2.4 , although this was while varying microphysics; simulations by Mason et al. (2009) produced a value of 1.58 in neutral conditions; and Anabor et al. (2011) produced a value of 1.34 in neutral conditions). It is not quite clear how universal this ratio is, as the maximum downdraft is clearly shown to depend on the ambient environment and the maximum outflow will depend on the environment as well as on variables such as surface roughness.

As downbursts develop and mature, the maximum wind speeds move with the ring vortex farther away from the downburst center. To show this, the downburst center is predicted as the location of maximum divergence over a roughly $1 \mathrm{~km}$ diameter area in both the $x$ and $y$ directions and is cal- 

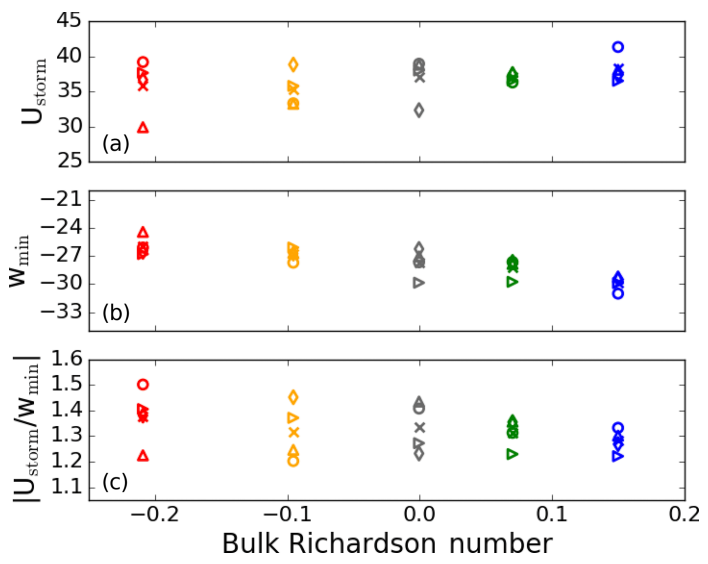

Figure 9. Variations of $U_{\text {storm }}(\mathbf{a}), w_{\min }(\mathbf{b})$, and the ratio between the two (c) for each case - C1 (red), C2 (yellow), N (gray), S1 (green), and S2 (blue) - with the average bulk Richardson number at the first model level over the first 3 min of simulation. Circles represent the $0 \mathrm{x} \_0 \mathrm{y}$ cases, the right-pointing triangles represent the $5 \mathrm{x} \_0 \mathrm{y}$ cases, the upward-pointing triangles represent the $0 \mathrm{x} \_5 \mathrm{y}$ cases, and the diamonds represent the 5x_5y cases. The ensemble mean is denoted by an " $\mathrm{x}$ " for each case.

culated every $15 \mathrm{~s}$. This process occasionally results in large jumps in the predicted downburst center location between model time steps; thus, in order to ameliorate the prediction, a linear regression is performed for the predicted centers between time burst $=6$ and $10.5 \mathrm{~min}$ and extrapolated to all times. Next, the maximum wind speeds at the lowest model level are recorded for each simulation in bins of $100 \mathrm{~m}$ expanding radially from the downburst center. In this way, we are able to track how the downburst expands and how the velocity field changes with radial distance from the center and with time as shown in Fig. 10. Here, only wind speeds to the right of the downburst center (downstream) are considered for the $\mathrm{C} 1$ (top row), $\mathrm{N}$ (middle row), and S2 (bottom row) cases with each ensemble member (columns 1 through 4) and the ensemble average (far right column) shown. It is clear that in each of the $\mathrm{C} 1$ simulations, the outflow begins to weaken earlier on in the simulations than for the neutral and S2 cases. C1-0x_5y, for example, appears to deteriorate just after reaching the surface. The length of time over which the outflow persists appears to be related to the ambient stability. The ensemble mean for the $\mathrm{S} 2$ case shows what appears to be a rather constant-intensity wind speed of around $30 \mathrm{~m} \mathrm{~s}^{-1}$ that persists past $12 \mathrm{~min}$ while the other cases show considerable weakening by this time. The propagation speed of the downbursts also appears to change with stability. In the $\mathrm{S} 2$ case, the peak wind speeds initially move away from the downburst center at a much quicker rate than for the other cases. However, after a minute or so, this propagation away from the center begins to slow to a pace similar to that of the $\mathrm{C} 1$ ensemble mean. Proctor (1989), being the only study in which a stable regime has been tested that we
Table 1. Ensemble-averaged maximum event horizontal wind speed, ensemble-averaged maximum event downdraft, and the ratio of those two for each case, along with the ensemble average of the time from first sign of divergence at the surface $\left(\nabla \cdot U_{\mathrm{sfc}}=\right.$ $\left.1.5 \nabla \cdot U_{\mathrm{sfc}_{0}}\right)$ to the time of maximum surface wind speed.

\begin{tabular}{lrrrrr}
\hline Case & $\begin{array}{r}U_{\text {storm }} \\
\left(\mathrm{m} \mathrm{s}^{-1}\right)\end{array}$ & $\begin{array}{r}w_{\text {min }} \\
\left(\mathrm{m} \mathrm{s}^{-1}\right)\end{array}$ & $\begin{array}{r}\mid U_{\text {storm }} / \\
w_{\min } \mid\end{array}$ & $\begin{array}{r}\text { Height of } \\
w_{\min }(\mathrm{km})\end{array}$ & $\begin{array}{r}t_{\text {ramp }} \\
(\min )\end{array}$ \\
\hline $\mathrm{C} 1$ & 35.86 & -25.98 & 1.38 & 0.371 & 1.54 \\
$\mathrm{C} 2$ & 35.30 & -26.82 & 1.32 & 0.343 & 2.44 \\
$\mathrm{~N}$ & 37.03 & -27.71 & 1.34 & 0.364 & 3.58 \\
$\mathrm{~S} 1$ & 37.02 & -28.22 & 1.31 & 0.364 & 3.07 \\
$\mathrm{~S} 2$ & 38.31 & -29.89 & 1.28 & 0.315 & 2.91 \\
\hline
\end{tabular}

are aware of, showed that a low-level temperature inversion acts to weaken the outflow wind speed, slow the propagation rate, and decrease the peak downdraft. In the simulations herein, the opposite occurs (see Table 1). This could be due to several factors: first, the depth of the stable layer is much shallower in these simulations (Proctor, 1989, considered an artificial temperature inversion through a depth of 500 and $1000 \mathrm{~m}$ ); second, the inclusion of low-level shear in these simulations appears to play a significant role in maintaining the ring vortex and, thus, generating a more persistent outflow; and third, Proctor (1989) utilizes a RANS model, whereas an LES model is utilized herein.

Upon studying the ensemble average for each case, it is clear that the $\mathrm{C} 1$ case increases to its peak velocity much quicker than the $\mathrm{N}$ and $\mathrm{S} 2$ cases. For each case, the time from which divergence at the lowest model level first increases past 0.5 times the average environmental surface divergence, $\nabla \cdot U_{\mathrm{sfc}}=1.5 \nabla \cdot U_{\mathrm{sfc}_{0}}$, to the time at which the maximum wind speed occurs, $t_{\max }$, is calculated and presented in Table 1 under $t_{\text {ramp. }}$. The variable, $t_{\text {ramp }}$, shows that the convective cases reach peak velocity quicker than in the neutral and stable cases, perhaps because the turbulence disorganizes the outflow more rapidly. However, it is interesting to note that the neutral case takes the longest time to reach peak velocity. On average, the $\mathrm{S} 2$ cases reach their peak velocity in less time than the S1 cases. We speculate that this is due to the ring vortex developing quicker with increased shear or potentially due to the additional negative buoyancy ingested from the surface-based stable layer.

\subsubsection{Ensemble-averaged variances}

Throughout each simulation, domain-averaged profiles of several resolved variances, $\sigma_{u_{i}}^{2}(t, z), \sigma_{v_{i}}^{2}(t, z)$, and $\sigma_{w_{i}}^{2}(t, z)$, are calculated and output every second for each ensemble member, $i$, in order to analyze how the downburst wind fields modify the environment. Note that with increased resolution, it is expected that the resolved variances will increase in the $\mathrm{S} 1$ and $\mathrm{S} 2$ cases. The resolution achieved in this model setup is adequate for convective and neutral simula- 


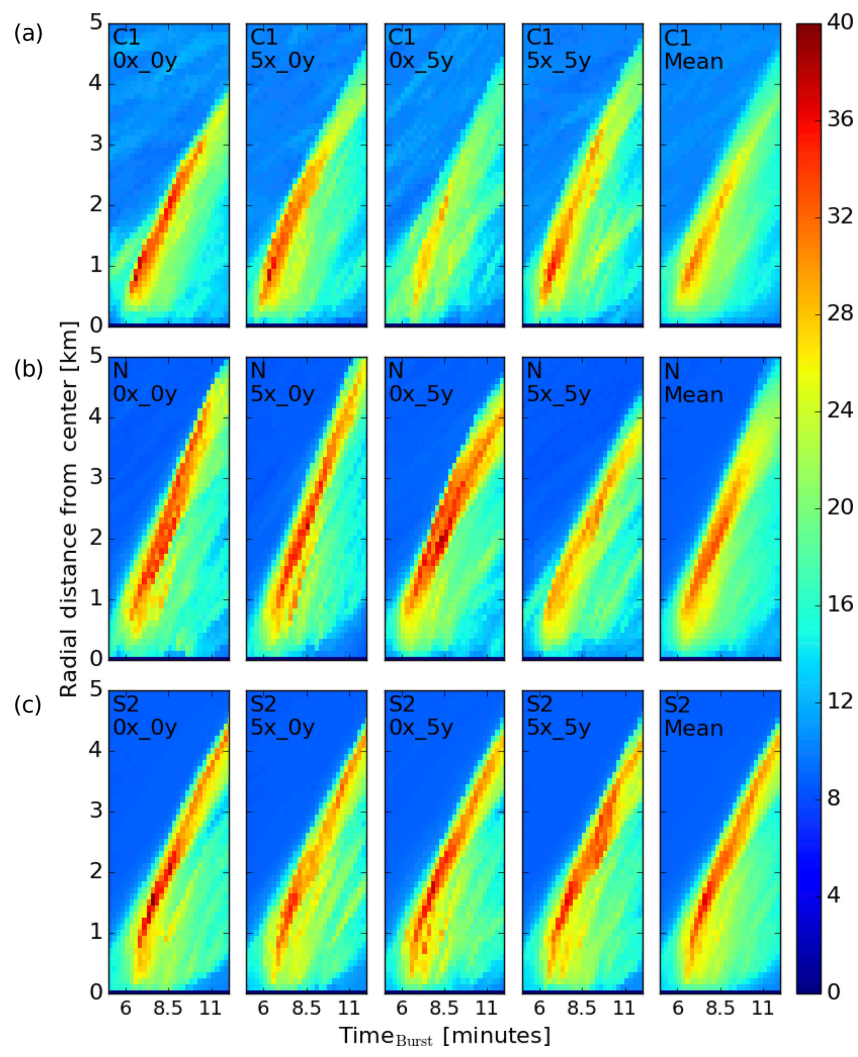

Figure 10. Variation with time of the horizontal wind speed $\left(\mathrm{m} \mathrm{s}^{-1}\right)$ at the lowest model level at binned radii to the right of the downburst center for cases $\mathrm{C} 1$ (a), N (b), and S2 (c). Each case is presented in the first four columns while the ensemble mean is shown in the right column.

tions. Next, the ensemble averages, $\left\langle\sigma_{u_{\text {Ens }}}^{2}\right\rangle(t, z),\left\langle\sigma_{v_{\text {Ens }}}^{2}\right\rangle(t, z)$, and $\left\langle\sigma_{w_{\text {Ens }}}^{2}\right\rangle(t, z)$, are computed for each case to get a generalized result. The profiles of maximum variance for the background (dotted) and the downburst (solid) are reported in Fig. 11. In terms of the environment for the maximum $u$ and $v$-component ensemble-averaged variances, the magnitude of these variances is seen to decrease with increasing stability. However, with the passage of the downburst, the peak variances occur near the surface and increase in magnitude as stability increases (note that due to the profiles being first domain-averaged, the variance generated by the downburst and the ambient environment are both included in these values). Both the $u$ - and $v$-component variances return very close to their ambient values by around $400 \mathrm{~m}$ and only truly begin to deviate and increase below $200 \mathrm{~m}$. The right panel shows the maximum of the ensemble-averaged vertical velocity variance, where a much more interesting feature is seen to develop. In the most convective case (C1), the maximum vertical velocity variance appears to increase while generally retaining the shape from the ambient profile. However, as the environment becomes more stable, a noselike profile begins to form. First, noticeable in $\mathrm{C} 2$, the nose
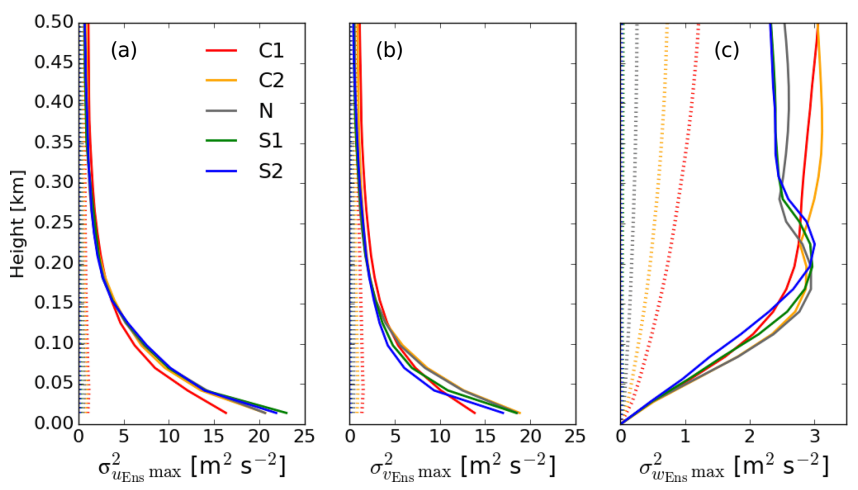

Figure 11. Profiles of the maximum $u, v$, and $w$ variance of the ensemble mean in panels $\mathbf{a}, \mathbf{b}, \mathbf{c})$, respectively, for each case. In each plot, for a given height, the maximum ensemble variance is computed over a specific time interval. In the case of the environment (dotted lines), the first $3 \mathrm{~min}$ of the output are considered, whereas, in the case of the downbursts, the first 13 min are utilized for the analysis.

increases in value (but not in height) to case N. As the stable layer develops, the peak begins to increase in height (but not much in value) from the $\mathrm{N}$ to the $\mathrm{S} 2$ case. Above this nose, the maximum begins to decrease as the environment becomes more stable. As opposed to the variances from the other two components of the wind field, the vertical velocity variance is the lowest at the surface, as one would expect, but grows significantly even with the influence of the ambient environment in the domain-averaged profile and reaches its peak value between around 160 and $225 \mathrm{~m}$. The combination of these variances being so large and their being so highly sheared within the lowest $200 \mathrm{~m}$ of the atmosphere further emphasizes the dangers of downburst winds to structures such as wind turbines.

In order to determine the cause of the nose-like structure in the maximum of the ensemble-averaged vertical velocity variance, Fig. 12 shows the variation with time of the ensemble mean of the vertical velocity variance profiles for each case. As in Fig. 8, open contours of negative (positive) vertical velocity in black (dotted magenta) are included for reference. At the time of the strongest downdraft, the ensembleaveraged vertical velocity variance is large for the convective cases but much weaker for the stable cases. This is due partly to the increased ambient vertical velocity variance in the convective cases, as can be seen in minutes $1-5$ of the simulations, as well as to the increased mixing from the cool downburst and warm thermals. As the outflow develops in each case, the vertical velocity variance shows a clear area of maximum variance coincident with the areas of large positive and negative vertical velocity. It is at this height that the nose-like feature exists and is due to the formation of the ring vortex. Further, it can be seen that the convective and neutral cases have heights of the area of maximum values that are around 160 to $175 \mathrm{~m}$, while the $\mathrm{S} 1$ and $\mathrm{S} 2$ cases 


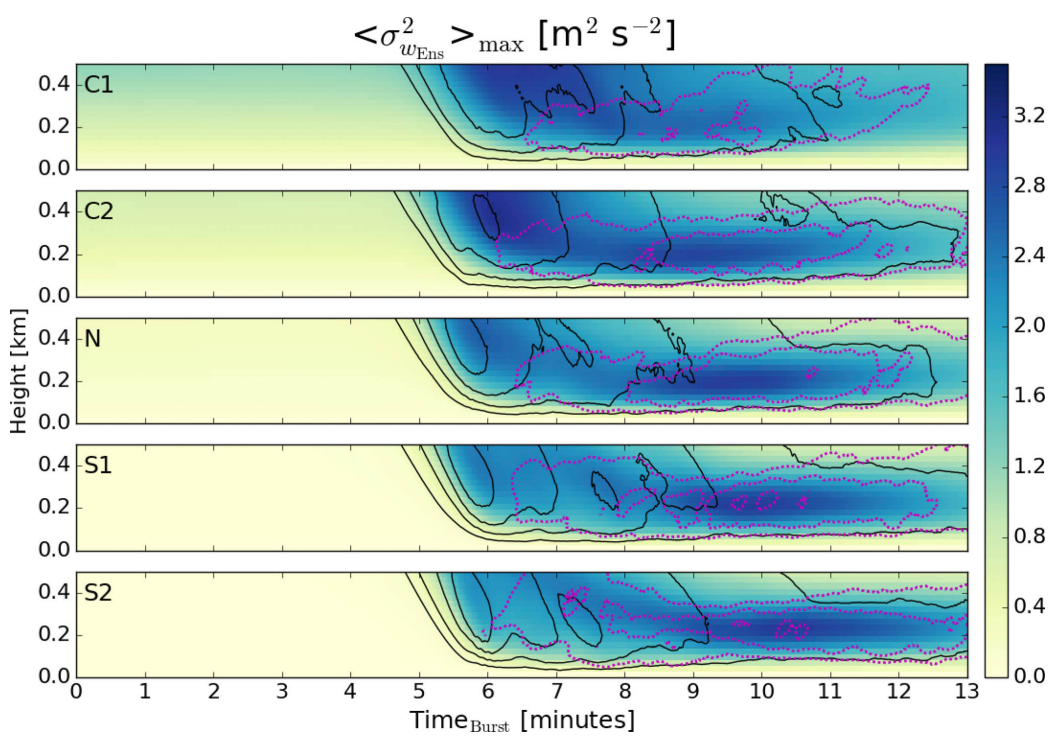

Figure 12. The same as Fig. 8 but for vertical velocity variance.

indicate that these heights are 200 and $225 \mathrm{~m}$, respectively. This also appears to be around the same heights as the top of the surface-based temperature inversion and low-level shear layers shown in Fig. 3, thus confirming results from Proctor (1989), where the height of the ring vortex is equivalent to that of the stable layer.

\subsubsection{Coherent turbulent kinetic energy}

Coherent, or organized, turbulent structures have been shown to increase structural loads and fatigue in wind turbines and have factored into decision-making for all aspects of the wind energy community from turbine design to siting and operation (Kelley et al., 2004, 2006). Kelley et al. (2004) and Kelley et al. (2006) define coherent turbulent kinetic energy, or CTKE, as a fluid dynamic parameter which describes both spatially and temporally organized motions in a turbulent field. While the primary focus for calculating CTKE has been for low-level jets and Kelvin-Helmholtz waves, here we compute the parameter from the organized turbulent motions of the downburst and associated ring vortex. The calculation of CTKE as defined in Kelley et al. (2004) is as follows:

$\mathrm{CTKE}=0.5 \sqrt{\left\langle u^{\prime} w^{\prime}\right\rangle^{2}+\left\langle u^{\prime} v^{\prime}\right\rangle^{2}+\left\langle v^{\prime} w^{\prime}\right\rangle^{2}}$,

where $\left\langle u^{\prime} w^{\prime}\right\rangle,\left\langle u^{\prime} v^{\prime}\right\rangle$, and $\left\langle v^{\prime} w^{\prime}\right\rangle$ are the domain averages of the kinematic momentum flux components. As an extension to the more conventional turbulent kinetic energy (TKE), CTKE effectively represents the amount of fluxes that are associated with organized structures. For example, in a completely random (homogeneous and isotropic) turbulent field, CTKE would be zero while TKE would be finite.

The total fluxes necessary to compute CTKE are output as domain-averaged profiles every second of the simulation.
Figure 13 shows the now familiar time-height plots with positive and negative vertical velocity contours for the ensembleaveraged value of CTKE for each case. Here we see that the convective cases generate the highest values of CTKE just as the downburst forms and strengthens. A trace of higher values of CTKE can also be seen at higher levels just after the maximum downdrafts occur and descending to where the ring vortex develops (most prominent in the neutral and stable cases). Interestingly, the simulations with the most welldefined ring vortices (S1 and S2) show the lowest values of CTKE at the levels in which the ring vortex exists. In each of the cases, the levels with the largest amounts of CTKE are in the lowest $150 \mathrm{~m}$. The convective cases have large amounts of CTKE through deeper levels; in contrast, case S1 shows a large amount of CTKE focused below $50 \mathrm{~m}$ and decreasing quickly above.

\subsubsection{Wavelet spectral analysis}

In order to quantify the energy at different scales, wavelet spectral analysis of the $u$-component wind field at $98.13 \mathrm{~m}$ is performed for each ensemble member of each case. The wavelet spectrum is computed from the time series data output every $1 \mathrm{~s}$ for each $y$ location using the Daubechies-5 (db5) wavelet. Sensitivity to the wavelet was performed with the Symmlet- 8 wavelet and the results showed very little dependence on the wavelet type (not shown). Due to the curvature of the outflow winds, the wind ramp does not occur for the selected vertical slice at the same time for all $y$. Thus, the wavelet spectra are averaged along $y$ between 4.5 and $5.5 \mathrm{~km}$ where the outflow reaches the vertical slice at roughly the same time. Further, at these points, as the outflow is almost directly to the east of the downburst center, the wind field is largely dominated by the $u$ component. Figure 14 shows 


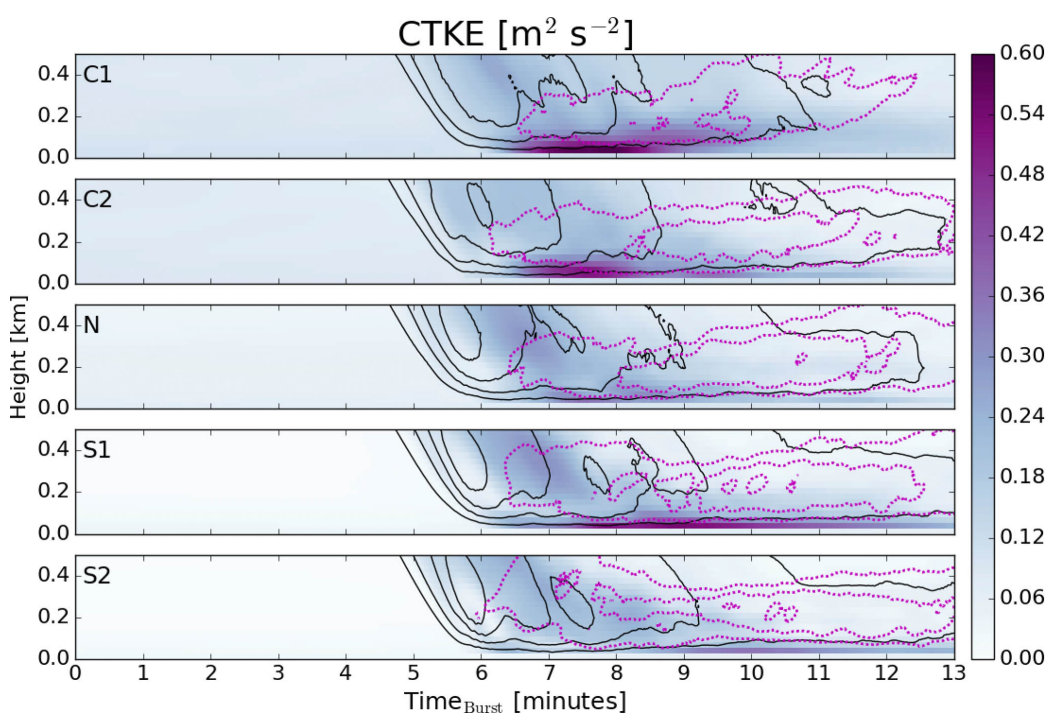

Figure 13. The same as Fig. 8 but for coherent turbulent kinetic energy (CTKE).

the resulting ensemble average of the wavelet power spectra for each case. The white portions of the figures are the areas inside of the "cone of influence", the region in which edge effects become important (Torrence and Compo, 1998). The non-stationary character of the turbulence associated with the $u$-component wind field is clear to see in each case. As would be expected, the ambient energy (from time ${ }_{\text {burst }}=2-6 \mathrm{~min}$ ) is higher for the convective cases and lower for the stable cases. In general, the lowest frequencies or small wavenumbers contain the peak in the wavelet spectra. At the time of the first impact of downbursts, energy increases by a factor of 10 or more across all the scales almost instantly. After this time, energy slowly declines across all frequencies. The convective cases resolve the highest amounts of energy at low frequencies compared to the other cases. As stability increases, the amount of energy resolved generally decreases; however, case $\mathrm{S} 1$ produces a fair amount more energy than the $\mathrm{N}$ and $\mathrm{S} 2$ cases. This same pattern is seen when studying CTKE (Fig. 13).

\section{Summary and conclusions}

In this study, a pseudo-spectral LES code is utilized to simulate several idealized downbursts during the ET. The ET is first simulated by spinning up a convective boundary layer and then linearly decreasing the surface temperature such that the boundary layer passes through a neutral regime and ultimately achieves a stable regime. The idealized downbursts are generated through a three-dimensional cooling source located over the top of the boundary layer to create a pocket of negatively buoyant air mimicking the latent cooling of evaporation, melting, and sublimation during a downburst. Downburst simulations are initialized separately within the various PBL regimes, allowing for the effects of stability to

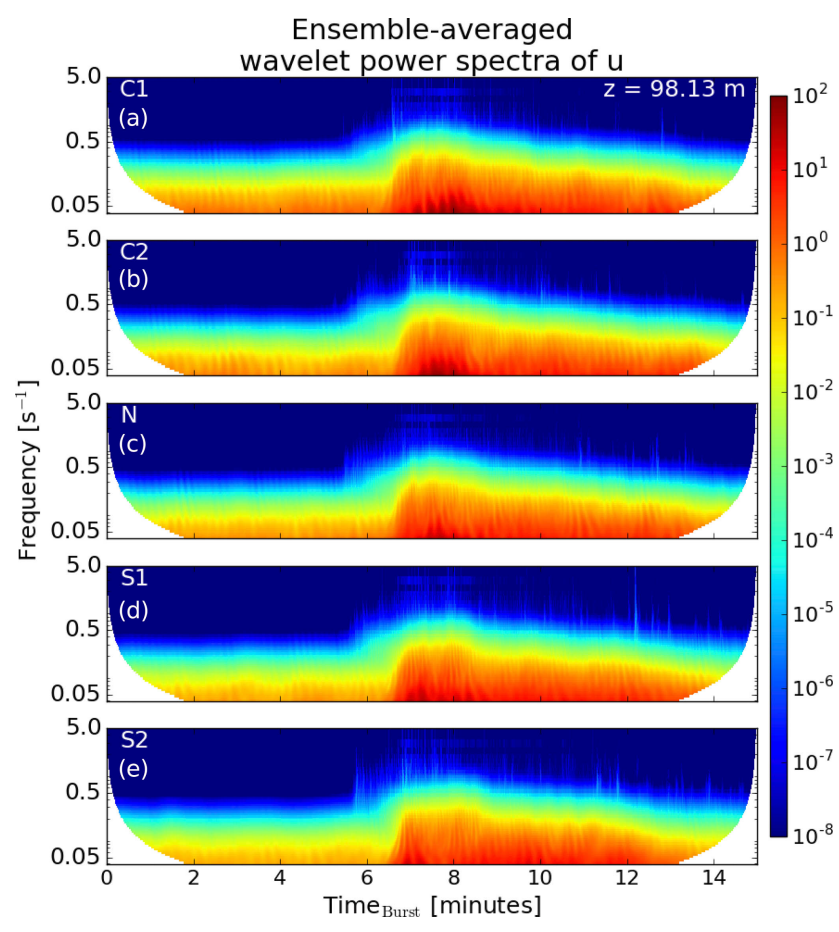

Figure 14. Ensemble-averaged wavelet power spectrum of $u$ component velocity $\left(\mathrm{m}^{2} \mathrm{~s}^{-1}\right)$ at $98.13 \mathrm{~m}$ using the Daubechies-5 wavelet for the $\mathrm{C} 1, \mathrm{C} 2, \mathrm{~N}, \mathrm{~S} 1$, and $\mathrm{S} 2$ cases from top to bottom, respectively.

be analyzed. Additionally, several instances are run for each stability regime by modifying the initial conditions in order to yield more generalized results. This is necessary due to the random locations of thermals in the convective boundary layer (and to a lesser extent, the neutral and stable boundary layers), which causes significant variation in the downburst 
winds. Analyses of these results allow for the following conclusions to be drawn:

- As seen with observed thunderstorm winds (see Fig. 1), the ensemble-averaged maximum wind speed remains fairly constant through each stability regime. However, the stable cases produce the most consistent and strongest outflow winds when compared to the convective and neutral cases. The consistency appears to be due to the increased homogeneity in the stable wind field and lack of ambient turbulence, while the increased severity is attributed to either a stronger ring vortex due to increased low-level shear, increased negative buoyancy generated from the vertical advection of the surface-based stable layer by the ring vortex, or a combination of the two.

- The maximum downdraft wind appears to show a direct positive correlation with increasing stability.

- The stronger ring vortex and associated stronger winds persist for a longer duration in the stable cases than in the convective and neutral cases due to the lack of turbulence to disorganize this feature. Further, the height of the ring vortex center increases with increasing stability, up to the height of the stable layer with the depth of the downburst head (based on temperature) becoming deeper in the stable cases.

- Maximum wind speeds are realized directly downstream from the center for the convective cases and north of the center for the stable cases; it appears that wind speeds reach their maxima where the outflow propagation direction is normal to the direction of the low-level wind shear vector. In the stable cases, the direction of the wind turns more southwesterly at the lowest levels, thus aligning the ambient wind shear vector with the northeast quadrant of the outflow winds.

- Calculations of CTKE show generally higher amounts of energy in the convective cases as the downburst first reaches the surface and spreads laterally. Wavelet analysis shows that this sudden increase in energy occurs across all scales as the downburst passes and then slowly begins to decline.

At the time of publication, a follow-up paper is currently in progress in which the output from these downburst simulations will be utilized as input into the FAST model. This work will aim to quantify the relationship downburst winds in various ambient stability regimes with the structural loads and stresses on commercial wind turbines.

Future work, motivated by the present study, includes a deeper investigation into the effects of stability on the dynamics of the downburst winds. Specifically, there is interest in isolating the effects of turbulence, low-level shear, and the surface-based temperature inversion in order to assess which boundary layer characteristics have the greatest impact on the downburst flow. Further, investigations into the relationship of outflow wind intensity and depth of the stable layer are planned; simulations by Proctor (1989) have shown that a deep enough stable layer can inhibit downburst winds and even prevent them from reaching the ground entirely. These studies will also allow the relationship between the depth of the stable layer and strength of the downburst in producing a heat burst to be determined. It is also of interest to investigate the accuracy of the current modeling framework for downbursts in various stability regimes. While it is reasonable to assume that the outflow winds from downbursts may encounter various stability regimes while advancing away from the downburst center, it is unclear to what extent the environment directly underneath the downburst is turbulent during the descent. Thus, future LES studies of the downburstproducing thunderstorm utilizing microphysics parameterizations are necessary to increase the realism of the simulations and to determine the representativeness of these findings.

Data availability. The simulated data (several terabytes in size) are freely available for any non-commercial research usage. They are archived in network-attached storage systems. Please contact the corresponding author for access.

Competing interests. The authors declare that they have no conflict of interest.

Acknowledgements. This work was supported by the National Science Foundation (NSF) under grant nos. CBET-1336760 and CBET-1336304.

Edited by: Joachim Peinke

Reviewed by: two anonymous referees

\section{References}

Anabor, V., Rizza, U., Nascimento, E. L., and Degrazia, G. A.: Large-Eddy Simulation of a microburst, Atmos. Chem. Phys., 11, 9323-9331, https://doi.org/10.5194/acp-11-9323-2011, 2011.

Anderson, J. R., Orf, L. G., and Straka, J. M.: A 3-D model system for simulating thunderstorm microburst outflows, Meteorol. Atmos. Phys., 49, 125-131, 1992.

Basu, S. and Porté-Agel, F.: Large-eddy simulation of stably stratified atmospheric boundary layer turbulence: A scale-dependent dynamic modeling approach, J. Atmos. Sci., 63, 2074-2091, 2006.

Basu, S., Vinuesa, J.-F., and Swift, A.: Dynamic LES modeling of a diurnal cycle, J. Appl. Meteorol. Clim., 47, 1156-1174, 2008.

Bowles, R. L. and Frost, W. (Eds.): Wind Shear/Turbulence Inputs to Flight Simulation and Systems Certification, NASA-CP-2474, Hampton, VA, 1987. 
Chay, M. T., Albermani, F., and Wilson, R.: Numerical and analytical simulation of downburst wind loads, Eng. Struct., 28, 240254, 2006.

Chen, L. and Letchford, C. W.: A deterministic-stochastic hybrid model of downbursts and its impact on a cantilevered structure, Eng. Struct., 26, 619-629, 2004.

Chen, X.: Analysis of alongwind tall building response to transient nonstationary winds, J. Struct. Eng., 134, 782-791, 2008.

Coffer, B. E. and Parker, M. D.: Impacts of increasing low-level shear on supercells during the early evening transition, Mon. Weather Rev., 143, 1945-1969, 2015.

Dahl, N. A., Nolan, D. S., Bryan, G. H., and Rotunno, R.: Using high-resolution simulations to quantify underestimates of tornado intensity from in situ observations, Mon. Weather Rev., 145, 1963-1982, 2017.

Doswell III, C. A. and Bosart, L. F.: Extratropical synoptic-scale processes and severe convection, in: Severe Convective Storms, 27-69, Springer, Boston, MA, 2001.

Doswell III, C. A., Brooks, H. E., and Dotzek, N.: On the implementation of the enhanced Fujita scale in the USA, Atmos. Res., 93, 554-563, 2009.

Edwards, R., LaDue, J. G., Ferree, J. T., Scharfenberg, K., Maier, C., and Coulbourne, W. L.: Tornado intensity estimation: Past, present, and future, B. Am. Meteor. Soc., 94, 641-653, 2013.

Fujita, T. T.: Proposed characterization of tornadoes and hurricanes by area and intensity, Tech. Rep. SMRP Research Paper 91, Deptartment of the Geophysical Sciences, University of Chicago, 1971.

Fujita, T. T.: Tornadoes and downbursts in the context of generalized planetary scales, J. Atmos. Sci., 38, 1511-1534, 1981.

Fujita, T. T.: The downburst, SMRP Research Paper Number 210, Department of the Geophysical Sciences, University of Chicago, 1985.

Fujita, T. T.: DFW microburst, SMRP Research Paper Number 217, Department of the Geophysical Sciences, University of Chicago, 1986.

Hawbecker, P., Basu, S., and Manuel, L.: Realistic simulations of the July 1, 2011 severe wind event over the Buffalo Ridge Wind Farm, Wind Energy, 20, 1803-1822, https://doi.org/10.1002/we.2122, 2017.

Holmes, J. D. and Oliver, S. E.: An empirical model of a downburst, Eng. Struct., 22, 1167-1172, 2000.

Jesson, M., Sterling, M., Letchford, C., and Baker, C.: Aerodynamic forces on the roofs of low-, mid- and high-rise buildings subject to transient winds, J. Wind Eng. Ind. Aerod., 143, 42-49, 2015.

Kelley, N. D., Shirazi, M., Jager, D., Wilde, S., Adams, J., Buhl, M., Sullivan, P., and Patton, E.: Lamar low-level jet project interim report, Tech. Rep. NREL/TP-500-34593, National Renewable Energy Laboratory, Golden, CO, 2004.

Kelley, N. D., Jonkman, B. J., and Scott, G. N.: The Great Plains turbulence environment: Its origins, impact and simulation, Tech. Rep. NREL/CP-500-40176, National Renewable Energy Laboratory, Golden, CO, 2006.

Kelly, D. L., Schaefer, J. T., and Doswell III, C. A.: Climatology of nontornadic severe thunderstorm events in the United States, Mon. Weather Rev., 113, 1997-2014, 1985.

Kessinger, C. J., Parsons, D. B., and Wilson, J. W.: Observations of a storm containing misocyclones, downbursts, and horizontal vortex circulations, Mon. Weather Rev., 116, 1959-1982, 1988.
Lundgren, T. S., Yao, J., and Mansour, N. N.: Microburst modelling and scaling, J. Fluid Mech., 239, 461-488, 1992.

Marshall, T. P.: Tornado damage survey at Moore, Oklahoma, Weather Forecast., 17, 582-598, 2002.

Mason, M. S., Wood, G. S., and Fletcher, D. F.: Numerical simulation of downburst winds, J. Wind Eng. Ind. Aerod., 97, 523-539, 2009.

Mason, M. S., Fletcher, D. F., and Wood, G. S.: Numerical simulation of idealised three-dimensional downburst wind fields, Eng. Struct., 32, 3558-3570, 2010a.

Mason, M. S., Wood, G. S., and Fletcher, D. F.: Numerical investigation of the influence of topography on simulated downburst wind fields, J. Wind Eng. Ind. Aerod., 98, 21-33, 2010 b.

McCarthy, J., Wilson, J. W., and Fujita, T. T.: The Joint Airport Weather Studies project, B. Am. Meteor. Soc., 63, 15-22, 1982.

McDonald, J. R. and Mehta, K. C.: A recommendation for an Enhanced Fujita scale (EF-Scale), Wind Science and Engineering Center, Texas Tech University, 2006.

Nguyen, H. H. and Manuel, L.: Thunderstorm downburst risks to wind farms, J. Renew. Sustain. Ener., 5, 013120, https://doi.org/10.1063/1.4792497, 2013.

Nguyen, H. H. and Manuel, L.: Extreme and fatigue loads on wind turbines during thunderstorm downbursts: The influence of alternative turbulence models, J. Renew. Sustain. Ener., 7, 013102, https://doi.org/10.1063/1.4905700, 2015.

Nguyen, H. H., Manuel, L., and Veers, P.: Simulation of inflow velocity fields and wind turbine loads during thunderstorm downbursts, in: 51st AIAA/ASME/ASCE/AHS/ASC Structures, Structural Dynamics, and Materials Conference, Orlando, FL, USA, 1-17, 2010.

Nguyen, H. H., Manuel, L., and Veers, P. S.: Wind turbine loads during simulated thunderstorm microbursts, J. Renew. Sustain. Ener., 3, 053104, https://doi.org/10.1063/1.3646764, 2011.

Nguyen, H. H., Manuel, L., Jonkman, J., and Veers, P. S.: Simulation of thunderstorm downbursts and associated wind turbine loads, J. Sol. Energ.-t ASME, 135, 021014, https://doi.org/10.1115/1.4023096, 2013.

Nieuwstadt, F. T. M. and Brost, R. A.: The decay of convective turbulence, J. Atmos. Sci., 43, 532-546, 1986.

Oliver, S. E., Moriarty, W. W., and Holmes, J. D.: A risk model for design of transmission line systems against thunderstorm downburst winds, Eng. Struct., 22, 1173-1179, 2000.

Oreskovic, C.: Numerical investigation of full scale thunderstorm downbursts: A parametric study and comparison to meteorological model, PhD thesis, University of Western Ontario, Canada, 2016.

Orf, L., Kantor, E., and Savory, E.: Simulation of a downburstproducing thunderstorm using a very high-resolution threedimensional cloud model, J. Wind Eng. Ind. Aerod., 104-106, 547-557, 2012.

Orf, L. G. and Anderson, J. R.: A numerical study of traveling microbursts, Mon. Weather Rev., 127, 1244-1258, 1999.

Orf, L. G., Anderson, J. R., and Straka, J. M.: A three-dimensional numerical analysis of colliding microburst outflow dynamics, J. Atmos. Sci., 53, 2490-2511, 1996.

Orf, L. G., Oreskovic, C., Savory, E., and Kantor, E.: Circumferential analysis of a simulated three-dimensional downburstproducing thunderstorm outflow, J. Wind Eng. Ind. Aerod., 135, 182-190, 2014. 
Park, J., Basu, S., and Manuel, L.: Large-eddy simulation of stable boundary layer turbulence and estimation of associated wind turbine loads, Wind Energy, 17, 359-384, 2014.

Proctor, F. H.: Numerical simulations of an isolated microburst. Part I: Dynamics and structure, J. Atmos. Sci., 45, 3137-3160, 1988.

Proctor, F. H.: Numerical simulations of an isolated microburst. Part II: Sensitivity experiments, J. Atmos. Sci., 46, 2143-2165, 1989.

Proctor, F. H.: Characteristics of a dry, pulsating microburst at Denver Stapleton Airport, Tech. Rep. 19950004156, NASA Langley Research Center, Hampton, VA, USA, 1994.

Reynolds, G. W.: Complications in estimating the magnitudes of tornado forces from damage analysis, in: 7th Conference on Severe Local Storms, American Meteorological Society, Kansas City, MO, 5-7, 1971.

Rotunno, R., Klemp, J. B., and Weisman, M. L.: A theory for strong, long-lived squall lines, J. Atmos. Sci., 45, 463-485, 1988.

Savory, E., Parke, G. A. R., Zeinoddini, M., Toy, N., and Disney, P.: Modelling of tornado and microburst-induced wind loading and failure of a lattice transmission tower, Eng. Struct., 23, 365-375, 2001.

Sengupta, A. and Sarkar, P. P.: Experimental measurement and numerical simulation of an impinging jet with application to thunderstorm microburst winds, J. Wind Eng. Ind. Aerod., 96, 345365,2008

Sengupta, A., Haan, F. L., Sarkar, P. P., and Balaramudu, V.: Transient loads on buildings in microburst and tornado winds, J. Wind Eng. Ind. Aerod., 96, 2173-2187, 2008.

Smith, M.: Visual observations of Kansas downbursts and their relation to aviation weather observations, Mon. Weather Rev., 114, 1612-1616, 1986.

Sorbjan, Z.: Decay of convective turbulence revisited, Bound.-Lay. Meteorol., 82, 501-515, 1997.

Srivastava, R. C.: A simple model of evaporatively driven downdraft: Application to microburst downdraft, J. Atmos. Sci., 42, 1004-1023, 1985.
Srivastava, R. C.: A model of intense downdrafts driven by the melting and evaporation of precipitation, J. Atmos. Sci., 44, 17521773, 1987.

Stull, R. B.: An Introduction to Boundary Layer Meteorology, Kluwer Academic Publishers: Dordrecht, the Netherlands, 670 pp., 1988.

Torrence, C. and Compo, G. P.: A practical guide to wavelet analysis, B. Am. Meteor. Soc., 79, 61-78, 1998.

Vermeire, B. C., Orf, L. G., and Savory, E.: Improved modelling of downburst outflows for wind engineering applications using a cooling source approach, J. Wind Eng. Ind. Aerod., 99, 801-814, 2011 a.

Vermeire, B. C., Orf, L. G., and Savory, E.: A parametric study of downburst line near-surface outflows, J. Wind Eng. Ind. Aerod., 99, 226-238, 2011b.

Vicroy, D. D.: A simple, analytical, axisymmetric microburst model for downdraft estimation, Tech. Rep. NASA-TM-104053, NASA, Hampton, VA, 1991.

Wakimoto, R. M.: Forecasting dry microburst activity over the high plains, Mon. Weather Rev., 113, 1131-1143, 1985.

Wakimoto, R. M.: Convectively driven high wind events, in: Severe Convective Storms, edited by: Doswell III, C. A., 255-298, Springer, Boston, MA, 2001.

Yao, J. and Lundgren, T. S.: Experimental investigation of microbursts, Exp. Fluids, 21, 17-25, 1996.

Zhang, Y., Hu, H., and Sarkar, P. P.: Modeling of microburst outflows using impinging jet and cooling source approaches and their comparison, Eng. Struct., 56, 779-793, 2013. 